DOI 10.4171/JEMS/418

Erwan Brugallé · Nicolas Puignau

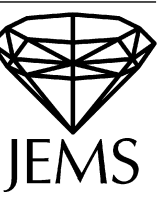

\title{
Enumeration of real conics and maximal configurations
}

Received June 17, 2011 and in revised form November 17, 2011

Abstract. We use floor decompositions of tropical curves to prove that any enumerative problem concerning conics passing through projective-linear subspaces in $\mathbb{R} P^{n}$ is maximal. That is, there exist generic configurations of real linear spaces such that all complex conics passing through these constraints are actually real.

Keywords. Tropical geometry, floor decomposition, real enumerative geometry, Gromov-Witten invariants

\section{Introduction}

A rational curve of degree $d$ in $\mathbb{C} P^{n}$ is parameterized by a polynomial map

$$
\phi: \mathbb{C} P^{1} \rightarrow \mathbb{C} P^{n}, \quad[t, u] \mapsto\left[P_{0}(t, u): \ldots: P_{n}(t, u)\right],
$$

where the $P_{i}(t, u)$ 's are homogeneous polynomials in two variables of degree $d$ with no common factors. Since Aut $\left(\mathbb{C} P^{1}\right)$ has dimension 3 and all the $P_{i}(t, u)$ 's are defined up to a common multiplicative constant, the dimension of the space of rational curves of degree $d$ in $\mathbb{C} P^{n}$ is

$$
(n+1)(d+1)-4=(n+1) d+(n-3) .
$$

Consequently, if we are looking for rational curves in $\mathbb{C} P^{n}$ satisfying exactly this number of independent conditions, we can reasonably expect the number of solution to be finite. For example, if $L$ is a linear subspace of codimension $j \geq 1$ in $\mathbb{C} P^{n}$, the condition "to intersect $L$ " imposes exactly $j-1$ independent conditions on rational curves in $\mathbb{C} P^{n}$. Hence, if we choose a generic configuration $\omega=\left\{L_{1}, \ldots, L_{\gamma}\right\}$ of linear subspaces of $\mathbb{C} P^{n}$, with $l_{j}=\operatorname{codim} L_{j} \geq 1$, such that

$$
\sum_{j=1}^{\gamma}\left(l_{j}-1\right)=(n+1) d+(n-3)
$$

E. Brugallé: Université Pierre et Marie Curie, Paris 6, 175 rue du Chevaleret, 75013 Paris, France; e-mail: brugalle@math.jussieu.fr

N. Puignau: Universidade Federal do Rio de Janeiro, Cidade Universitária - Ilha do Fundão, 21941-909 Rio de Janeiro, Brasil; e-mail: puignau@im.ufrj.br

Mathematics Subject Classification (2010): Primary 14N10, 14P05; Secondary 14N35, 14N05 
we expect a finite number of rational curves of degree $d$ in $\mathbb{C} P^{n}$ intersecting all the linear subspaces in $\omega$. The term "generic" means that these linear subspaces have to be chosen so that they impose altogether independent conditions.

It turns out that this number of rational curves, which we denote by $N_{d, n}\left(l_{1}, \ldots, l_{\gamma}\right)$, is indeed finite and does not depend on the configuration $\omega$ we have chosen, but only on $n$, $d, l_{1}, \ldots, l_{\gamma}$. The numbers $N_{d, n}\left(l_{1}, \ldots, l_{\gamma}\right)$ are known as Gromov-Witten invariants of the projective space $\mathbb{C} P^{n}$. For example, since there exists a unique line passing through two distinct points in $\mathbb{C} P^{n}$, we have

$$
N_{1, n}(n, n)=1 \quad \forall n \geq 2 .
$$

For a more detailed introduction to Gromov-Witten theory, we refer the interested reader to the excellent book [KV06]. In this paper, it is convenient to extend the definition of the numbers $N_{d, n}\left(l_{1}, \ldots, l_{\gamma}\right)$ to any set of $\gamma$ numbers in $\mathbb{Z}$ by

$N_{d, n}\left(l_{1}, \ldots, l_{\gamma}\right)=0 \quad$ if $\sum_{j=1}^{\gamma}\left(l_{j}-1\right) \neq(n+1) d+(n-3)$ or $\exists j, l_{j} \leq 0$ or $l_{j} \geq n+1$.

All linear spaces in our generic configuration $\omega$ can be chosen to be real. In this case, it makes sense to enumerate real rational curves in $\mathbb{C} P^{n}$ (i.e. rational curves which are invariant under the complex conjugation of $\mathbb{C} P^{n}$ ) of degree $d$ intersecting our configuration of real linear subspaces. Unlike the enumeration of complex curves, the number of real solutions, denoted by $N_{d, n}^{\mathbb{R}}\left(l_{1}, \ldots, l_{\gamma}, \omega\right)$, now depends on the chosen configuration $\omega$ of linear spaces. Clearly, we have the inequality

$$
N_{d, n}^{\mathbb{R}}\left(l_{1}, \ldots, l_{\gamma}, \omega\right) \leq N_{d, n}\left(l_{1}, \ldots, l_{\gamma}\right) \quad \forall \omega .
$$

However, it is unknown in general if there exists a real configuration $\omega$ such that all complex solutions are real. For example, can the 92 complex conics passing through eight general lines in $\mathbb{R} P^{3}$ be real? More generally, it is an important and difficult question to ask how many solutions of an enumerative problem can be real (see [Ful84, §7.2]). When all complex solutions can be real, we say that this enumerative problem is maximal.

To stress how difficult these questions are, let us summarize the very few things known in 2011 about the maximality of the enumerative problems defined above. Since the corresponding Gromov-Witten invariant is equal to 1, it is trivial that the problem is maximal in the following two cases:

- $d=1, l_{j}=n$ for some $j$;

- $n=2, d=2$, and $l_{1}=l_{2}=l_{3}=l_{4}=l_{5}=2$.

It is also easy to see that the problem is maximal in the case $n=2$ and $d=3$ (and so $l_{1}=\cdots=l_{8}=2$ ). The first systematic non-trivial result was obtained by Sottile who proved in [Sot97] that the problem is maximal as soon as $d=1$ (the so-called problems of "Schubert-type"; actually problems of Schubert-type involving linear subspaces of any dimension turn out to be maximal by [Vak06]). More recently, with the help of tropical geometry, it was proved in [BM07] that the problem above is maximal for $d=2$ and $n=3$. Up to our knowledge, nothing more was known before our investigation. 
Our main result is that the enumerative problems discussed above are maximal when $d=2$. Theorem 1.1 is a direct consequence of Theorem 2.5, Lemma 2.6, and Proposition 4.5 .

Theorem 1.1. For $n \geq 2, l_{1} \geq 1, \ldots, l_{\gamma} \geq 1$ satisfying

$$
\sum_{j=1}^{\gamma}\left(l_{j}-1\right)=3 n-1,
$$

there exists a generic configuration $\omega=\left\{L_{1}, \ldots, L_{\gamma}\right\}$ of real linear subspaces of $\mathbb{C} P^{n}$ such that $\operatorname{codim} L_{j}=l_{j}$ and

$$
N_{2, n}^{\mathbb{R}}\left(l_{1}, \ldots, l_{\gamma}, \omega\right)=N_{2, n}\left(l_{1}, \ldots, l_{\gamma}\right) .
$$

To prove Theorem 1.1, we use floor decomposition of tropical curves. In his pioneering work [Mik05], Mikhalkin reduced the enumeration of complex and real algebraic curves in $\left(\mathbb{C}^{*}\right)^{2}$ to the enumeration of some piecewise linear graphs in $\mathbb{R}^{2}$ called plane tropical curves. Shortly afterwards these results were extended in [Mik] and [NS06] to the computation of genus 0 Gromov-Witten invariants of projective spaces of arbitrary dimension. By stretching configurations of constraints along some specific direction, Brugallé and Mikhalkin [BM] replaced the enumeration of tropical curves by a purely combinatorial study of their floor decompositions. As an application, they exhibited a generic configuration of eight real lines in $\mathbb{R} P^{3}$ with 92 real conics passing through them.

In this paper, we refine the technique used in [BM07] in the case of $\mathbb{C} P^{3}$ to systematically study the case $d=2$. Along the way, we will give a proof of Sottile's Theorem different from the original one.

The question of existence of non-trivial lower bounds for the numbers $N_{2, n}^{\mathbb{R}}\left(l_{1}, \ldots\right.$, $\left.l_{\gamma}, \omega\right)$ is also an important and difficult problem about which not much is known. The combination of Welschinger invariants (see [Wel05a] and [Wel05b]) and tropical geometry allowed exhibiting such non-trivial lower bounds in the case of rational curves passing through points in $\mathbb{R} P^{2}$ or $\mathbb{R} P^{3}$, i.e. for $n=2$ or 3 and $l_{i}=n$ for all $i$ (see [Wel05a], [Mik05], and [IKS04] for the case $n=2$, and [Wel05b], and [BM] for the case $n=3$ ). In the case of enumeration of lines (and more generally in the enumeration of real linear spaces), the existence of some non-trivial lower bounds has been proved by Gabrielov and Eremenko in [EG02]. Up to our knowledge, the exact minimal value of $N_{d, n}^{\mathbb{R}}\left(l_{1}, \ldots, l_{\gamma}, \omega\right)$ (when non-trivial) is known so far only in the cases $n=2, d=3$ ([DK00]) and $d=4$ ([Rey]), and in the cases $d=1$ and $l_{i}=2$ for all $i$ ([EG02]).

One could also study maximality of more general real enumerative problems, for example by prescribing tangency conditions with constraints. We refer the interested reader to [RTV97], [Ber08], [Sot], and [BBM] for some partial answers in this direction.

In Section 2 we give all tropical definitions needed to prove Theorem 1.1. In particular, we set up the tropical enumerative problems studied in this paper. Next, in Section 3 we explain the main ideas of the floor decomposition technique to solve these tropical enumerative problems, before focusing on the easier particular cases of enumeration of lines and conics. Theorem 1.1 is finally proved in Section 4. 


\section{Tropical geometry}

In this section, we briefly review the tropical background needed in this paper. For more details, we refer the reader, for example, to [Mik06], [RGST05], [IMS07], and [BPS08].

\subsection{Rational tropical curves}

Given a finite graph $C$ (i.e. $C$ has a finite number of edges and vertices) we denote by $\operatorname{Vert}^{\infty}(C)$ (resp. Vert $\left.{ }^{0}(C)\right)$ the set of vertices of $C$ which are (resp. are not) 1-valent, and by Edge ${ }^{\infty}(C)$ (resp. Edge ${ }^{0}(C)$ ) the set of edges which are (resp. are not) adjacent to a 1 -valent vertex. Throughout the text, we will always assume that the graphs considered have no 2-valent vertices.

Definition 2.1. A rational tropical curve $C$ is a finite compact connected tree equipped with a complete inner metric on $C \backslash \operatorname{Vert}^{\infty}(C)$.

By definition, the 1-valent vertices of $C$ are at infinite distance from all the other points of $C$. The elements of Edge ${ }^{\infty}(C)$ are called the ends of $C$. An edge in Edge ${ }^{\infty}(C)$ (resp. Edge ${ }^{0}(C)$ ) is said to be unbounded (resp. bounded).

Example 1. An example of a rational tropical curve $C$ is depicted in Figure 1. This curve has five unbounded edges, and two bounded edges of finite lengths $a$ and $b$. All the edge lengths are indicated in the figure.

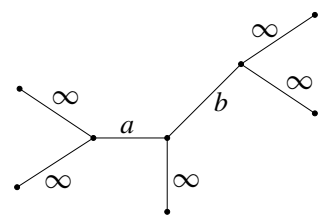

Fig. 1. A rational tropical curve.

Given $e$ an edge of a tropical curve $C$, we choose a point $p$ in the interior of $e$ and a unit vector $u_{e}$ of the tangent line to $C$ at $p$. Of course, the vector $u_{e}$ depends on the choice of $p$ and is well-defined only up to multiplication by -1 , but this will not matter in the following. We will sometimes need $u_{e}$ to have a prescribed direction, and we will then specify it. The standard inclusion of $\mathbb{Z}^{n}$ in $\mathbb{R}^{n}$ induces a standard inclusion of $\mathbb{Z}^{n}$ in the tangent space of $\mathbb{R}^{n}$ at any point of $\mathbb{R}^{n}$.

Definition 2.2. Let $C$ be a rational tropical curve. A continuous map $f: C \backslash \operatorname{Vert}^{\infty}(C)$ $\rightarrow \mathbb{R}^{n}$ is a tropical morphism if

- for any edge $e$ of $C$, the restriction $f_{\mid e}$ is a smooth map with $d f\left(u_{e}\right)=w_{f, e} u_{f, e}$ where $u_{f, e} \in \mathbb{Z}^{n}$ is a primitive vector, and $w_{f, e}$ is a non-negative integer;

- for any vertex $v$ in $\operatorname{Vert}^{0}(C)$ whose adjacent edges are $e_{1}, \ldots, e_{k}$, one has the balancing condition

$$
\sum_{i=1}^{k} w_{f, e_{i}} u_{f, e_{i}}=0
$$

where $u_{e_{i}}$ is chosen so that it points away from $v$. 
The integer $w_{f, e}$ is called the weight of the edge $e$ with respect to $f$. When no confusion is possible, we speak about the weight of an edge, without referring to the morphism $f$. By abuse of notation, we write $f: C \rightarrow \mathbb{R}^{n}$ instead of $f: C \backslash \operatorname{Vert}^{\infty}(C) \rightarrow \mathbb{R}^{n}$. If $w_{f, e}=0$, we say that the morphism $f$ contracts the edge $e$. The morphism $f$ is called minimal if it does not contract any edge.

Given a vector $u=\left(u_{1}, \ldots, u_{n}\right)$ in $\mathbb{R}^{n}$, we define

$$
d_{u}=\max \left\{0, u_{1}, \ldots, u_{n}\right\}
$$

The degree of a tropical morphism $f: C \rightarrow \mathbb{R}^{n}$ is defined by

$$
\sum_{e \in \operatorname{Edge}^{\infty}(C)} w_{f, e} d_{u_{f, e}}
$$

where $u_{f, e}$ is chosen so that it points to its adjacent 1 -valent vertex.

We define the following vectors in $\mathbb{R}^{n}: U_{1}=(-1,0, \ldots, 0), U_{2}=(0,-1,0, \ldots, 0)$, $\ldots, U_{n}=(0, \ldots, 0,-1)$, and $U_{n+1}=(1, \ldots, 1)$. A tropical morphism $f: C \rightarrow \mathbb{R}^{n}$ of degree $d$ is said to be transverse at infinity if $C$ has exactly $(n+1) d$ non-contracted ends. Note that in this case, for any $i=1, \ldots, n+1$, the curve $C$ has exactly $d$ edges $e \in$ Edge $^{\infty}(C)$ with $u_{f, e}=U_{i}$, where $u_{f, e}$ is chosen so that it points to its adjacent 1 -valent vertex.

Example 2. Figure 2 depicts a tropical conic in $\mathbb{R}^{2}$ and a tropical conic in $\mathbb{R}^{3}$. For each unbounded edge $e$, the vector $u_{f, e}$ pointing to infinity is given next to $e$.
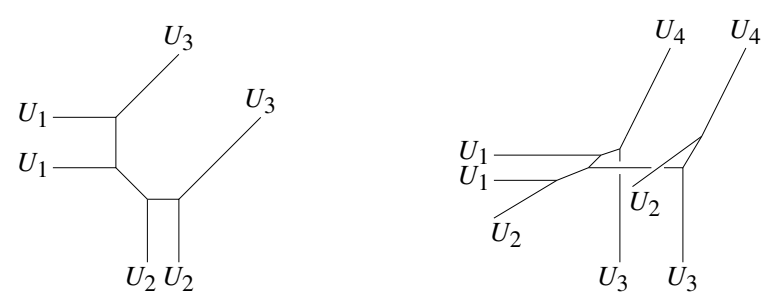

Fig. 2. Tropical conics in $\mathbb{R}^{2}$ and in $\mathbb{R}^{3}$.

Two tropical morphisms $f_{1}: C_{1} \rightarrow \mathbb{R}^{n}$ and $f_{2}: C_{2} \rightarrow \mathbb{R}^{n}$ are said to be isomorphic if there exists an isomorphism of metric graphs $\phi: C_{1} \rightarrow C_{2}$ such that $f_{1}=f_{2} \circ \phi$. In this text, we consider tropical curves and tropical morphisms up to isomorphism.

Two tropical morphisms $h: C_{1} \rightarrow \mathbb{R}^{n}$ and $h^{\prime}: C_{2} \rightarrow \mathbb{R}^{n}$ are said to be of the same combinatorial type if there exists a homeomorphism of graphs $\phi: C_{1} \rightarrow C_{2}$ (i.e. we forget about the metric on $C_{1}$ and $C_{2}$ ) such that $h=h^{\prime} \circ \phi$, and $w_{h, e}=w_{h^{\prime}, \phi(e)}$ for all $e \in \operatorname{Edge}\left(C_{1}\right)$.

In this text, we need the notion of reducible tropical morphism. Given two tropical curves $C_{1}$ and $C_{2}$, two points $p_{1}$ and $p_{2}$ respectively on $C_{1}$ and $C_{2}$, the topological gluing $C_{1} \cup \cup_{\left(p_{1}, p_{2}\right)} C_{2}$ of $C_{1}$ and $C_{2}$ at $p_{1}$ and $p_{2}$ inherits naturally a structure of tropical curve from $C_{1}$ and $C_{2}$. The curve $C_{i}$ can be seen as a subset of $C$, and the point $p_{1}=p_{2}$ is called the node of $C$. 
Definition 2.3. A minimal tropical morphism $f: C \rightarrow \mathbb{R}^{n}$ is said to be reducible if there exist two minimal tropical morphisms $f_{1}: C_{1} \rightarrow \mathbb{R}^{n}$ and $f_{2}: C_{2} \rightarrow \mathbb{R}^{n}$, and points $p_{i} \in C_{i}$, such that $C$ is the gluing of $C_{1}$ and $C_{2}$ at the points $p_{1}$ and $p_{2}$, and $f_{\mid C_{i}}=f_{i}$.

We denote such a reducible tropical morphism $f=f_{1} \cup_{p} f_{2}: C_{1} \cup_{p} C_{2} \rightarrow \mathbb{R}^{n}$. If $f: C \rightarrow \mathbb{R}^{n}$ is a reducible tropical morphism of degree $d$, then $f_{i}: C_{i} \rightarrow \mathbb{R}^{n}$ is a tropical morphism of degree $d_{i}$ and $d_{1}+d_{2}=d$. In particular, if $d=2$ then $d_{1}=d_{2}=1$.

\subsection{Tropical linear spaces}

Defining tropical linear spaces of $\mathbb{R}^{n}$ in full generality would require much more material than needed in the rest of the paper. Moreover this would force us to make the distinction between realizable and non-realizable tropical linear spaces, a notion we want to keep out of the scope of this note. Instead, we define a restricted class of tropical linear spaces of $\mathbb{R}^{n}$, which we call complete tropical linear spaces. For a general definition and study of tropical linear spaces, we refer to [Spe08].

Given $1 \leq i<j \leq n+1$, we denote by $E_{i, j}$ the convex polyhedron of $\mathbb{R}^{n}$ obtained by taking all non-negative real linear combinations of all the vectors $U_{k}$ but $U_{i}$ and $U_{j}$, and we define

$$
H_{0}^{n}=\bigcup_{1 \leq i<j \leq n+1} E_{i, j}
$$

Definition 2.4. A tropical hyperplane of $\mathbb{R}^{n}$ is the translation of $H_{0}^{n}$ along any vector of $\mathbb{R}^{n}$.

A complete tropical linear space of dimension $j$ is the intersection of $n-j$ tropical hyperplanes in general position. The ambient space $\mathbb{R}^{n}$ is a complete tropical linear space of dimension $n$.

One could avoid the genericity assumption in Definition 2.4 by considering tropical (or stable) intersections of tropical hyperplanes in $\mathbb{R}^{n}$. We refer to [Mik06] or [RGST05] for more details.

A tropical linear space of dimension $j$ is a finite polyhedral complex of pure dimension $j$.

Example 3. A tropical plane and a tropical line in $\mathbb{R}^{3}$ are depicted in Figure 3.
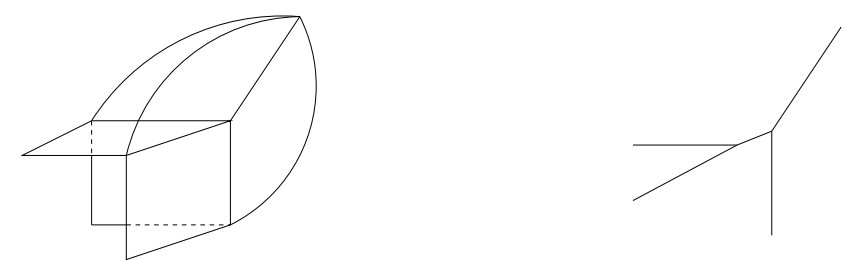

Fig. 3. A tropical plane and a tropical line in $\mathbb{R}^{3}$. 


\subsection{Tropical enumerative geometry}

Now that we have defined tropical rational curves and complete tropical linear spaces in $\mathbb{R}^{n}$, we can play the same game as in Section 1 . Namely, let us fix some integers $d \geq 1, n \geq 2, \gamma \geq 2, l_{1} \geq 1, \ldots, l_{\gamma} \geq 1$ subject to equality (1), and let us choose a configuration $\omega=\left\{L_{1}, \ldots, L_{\gamma}\right\}$ of complete tropical linear spaces in $\mathbb{R}^{n}$ such that $\operatorname{codim} L_{j}=l_{j}$ for $j=1, \ldots, \gamma$. Then we define $\mathbb{T C}(\omega)$ as the set of all minimal rational morphisms $f: C \rightarrow \mathbb{R}^{n}$ of degree $d$ such that $f(C)$ intersects all tropical linear spaces in $\omega$. This game is related to Section 1 by the following fundamental theorem.

Theorem 2.5 (Correspondence Theorem, [Mik05], [Mik], [NS06]). If $\omega$ is generic, then the set $\mathbb{T C}(\omega)$ is finite and composed of tropical morphisms transverse to infinity. Moreover, to each element $f$ in $\mathbb{T C}(\omega)$, one can associate a positive integer number $\mu(f)$, called the multiplicity of $f$, which depends only on $f$ and $\omega$, such that

$$
N_{d, n}\left(l_{1}, \ldots, l_{\gamma}\right)=\sum_{f \in \mathbb{T C}(\omega)} \mu(f) .
$$

Proof. As explained in [BBM, Section 6] the multiplicity of $f$ reduces to local computations, which are done in [NS06].

There is a combinatorial definition of the integer $\mu(f)$ just in terms of the tropical morphism $f$ and the configuration $\omega$. However we will not need it in this paper, so instead of giving the precise definition of $\mu(f)$, let us just explain its geometrical meaning.

Theorem 2.5 is obtained by degenerating the standard complex structure on $\left(\mathbb{C}^{*}\right)^{n}$ via the following self-diffeomorphism of $\left(\mathbb{C}^{*}\right)^{n}$ :

$$
H_{t}:\left(\mathbb{C}^{*}\right)^{n} \rightarrow\left(\mathbb{C}^{*}\right)^{n}, \quad\left(z_{i}\right) \mapsto\left(\left|z_{i}\right|^{\frac{1}{\log t}} \frac{z_{i}}{\left|z_{i}\right|}\right),
$$

Namely, for any tropical complete linear space $L_{j}$ of codimension $l_{j}$ in $\omega$, there exists a family $\left(L_{t, j}\right)_{t>0}$ of complex linear spaces in $\left(\mathbb{C}^{*}\right)^{n}$ of codimension $l_{j}$ such that the set $\log \circ H_{t}\left(L_{t, j}\right)$ converges to $L_{j}$ when $t \rightarrow \infty$, in the Hausdorff metric on compact subsets of $\left(\mathbb{R}^{*}\right)^{n}$. The map $\log$ is defined by $\log \left(z_{i}\right)=\left(\log \left|z_{i}\right|\right)$. Hence to each $t$, we associate a configuration $\omega_{t}=\left\{L_{t, 1}, \ldots, L_{t, \gamma}\right\}$ of linear subspaces of $\left(\mathbb{C}^{*}\right)^{n}$. For $t$ large enough, the configuration $\omega_{t}$ is generic if $\omega$ is generic, so the complex rational curves of degree $d$ passing through all the linear spaces in $\omega_{t}$ form a finite $\operatorname{set} \mathcal{C}\left(\omega_{t}\right)$. It turns out, and this is the core of Theorem 2.5, that the set $\log \circ H_{t}\left(\mathcal{C}\left(\omega_{t}\right)\right)$ converges to the set $\mathbb{T C}(\omega)$, and that for any tropical morphism $f \in \mathbb{C} C(\omega)$ there exist exactly $\mu(f)$ complex curves in $\mathcal{C}\left(\omega_{t}\right)$ whose image under $\log \circ H_{t}$ converge to $f(C)$.

Suppose now that the linear spaces $L_{t, j}$ are chosen to be all real (this is always possible). In particular, all curves in $\mathcal{C}\left(\omega_{t}\right)$ are either real or come in pairs of complex conjugate curves. A very important property of the map $H_{t}$ is that it commutes with the standard complex conjugation in $\left(\mathbb{C}^{*}\right)^{n}$. As a consequence, both curves in a pair of complex conjugate curves in $\mathcal{C}\left(\omega_{t}\right)$ have the same image under $\log \circ H_{t}$. In particular we have the following lemma, where $[\mu(f)]_{2}$ denotes the value modulo 2 of the integer $\mu(f)$. 
Lemma 2.6. If $\omega$ is generic, then there exists a generic configuration $\Omega$ of real linear spaces in $\mathbb{R} P^{n}$ such that there exist at least $\sum_{f \in \mathbb{C} C(\omega)}[\mu(f)]_{2}$ real rational curves of degree $d$ in $\mathbb{R} P^{n}$ intersecting all linear spaces in $\Omega$.

Theorem 1.1 is a consequence of Theorem 2.5 and Lemma 2.6: we exhibit generic configurations $\omega$ such that the set $\mathbb{T C}(\omega)$ contains exactly $N_{2, n}\left(l_{1}, \ldots, l_{\gamma}\right)$ distinct tropical curves. Hence, all of them must have multiplicity 1 , which implies the maximality of the corresponding enumerative problem by Lemma 2.6. The main tool to exhibit such configurations $\omega$ is the floor decomposition technique.

\subsection{Enumeration of tropical reducible conics}

Here we state some easy facts about a small variation of the problem presented in Section 2.3. Namely, we enumerate tropical reducible conics passing through a generic collection of complete tropical linear spaces.

The next lemma is standard (see for example [BBM] or [GKM09]).

Lemma 2.7. Let $\alpha$ be a combinatorial type of reducible morphisms $f_{1} \cup_{p} f_{2}: C_{1} \cup_{p} C_{2}$ $\rightarrow \mathbb{R}^{n}$ of degree 2 . Then the space of all reducible tropical morphisms with combinatorial type $\alpha$ is naturally a convex polyhedron of dimension at most $3 n-2$, with equality if and only if $C_{i}$ is trivalent and $p_{i}$ is not a vertex of $C_{i}$ for $i=1,2$.

Let us fix some integers $l_{0} \geq 0$ and $l_{1}^{1}, \ldots, l_{\gamma_{1}}^{1}, l_{1}^{2}, \ldots, l_{\gamma_{2}}^{2} \geq 1$ such that

$$
l_{0}+\sum_{i=1}^{2} \sum_{j=1}^{\gamma_{i}}\left(l_{j}^{i}-1\right)=3 n-2
$$

and let us choose a tropical complete linear space $L_{0}$ in $\mathbb{R}^{n}$ of codimension $l_{0}$ and two configurations $\omega^{1}$ and $\omega^{2}$ of complete tropical linear spaces in $\mathbb{R}^{n}$ such that $\omega^{i}=$ $\left\{L_{1}^{i}, \ldots, L_{\gamma_{i}}^{i}\right\}$ with $\operatorname{codim} L_{j}^{i}=l_{j}^{i}$. Then we define $\mathbb{T} \mathcal{C}_{\text {red }}\left(L_{0}, \omega^{1}, \omega^{2}\right)$ as the set of all reducible rational morphisms $f=f_{1} \cup_{p} f_{2}: C_{1} \cup_{p} C_{2} \rightarrow \mathbb{R}^{n}$ of degree 2 such that $f_{i}\left(C_{i}\right)$ intersects all tropical linear spaces in $\omega^{i}$ for $i=1,2$ and $f(p) \in L_{0}$. Note that if $\mathbb{T} \mathcal{C}_{\text {red }}\left(L_{0}, \omega^{1}, \omega^{2}\right) \neq \emptyset$, we necessarily have

$$
\sum_{j=1}^{\gamma_{i}}\left(l_{j}^{i}-1\right) \leq 2 n-2 \quad \text { for } i=1,2 .
$$

The next lemma is a straightforward application of Lemma 2.7 and standard techniques in tropical enumerative geometry (see for example [BBM], [NS06], or [GKM09]).

Lemma 2.8. For a generic choice of $L_{0}, \omega^{1}$, and $\omega^{2}$, the set $\mathbb{T} \mathcal{C}_{\text {red }}\left(L_{0}, \omega^{1}, \omega^{2}\right)$ is finite, and composed of tropical morphisms transverse to infinity.

Note that we can pose the same problem in complex geometry, and that we can easily give the answer in terms of the numbers $N_{1, n}$. Namely, let $L_{0}$ be a linear space in $\mathbb{C} P^{n}$ of codimension $l_{0}$, and $\omega^{1}$ and $\omega^{2}$ two configurations of linear spaces in $\mathbb{C} P^{n}$ such that $\omega^{i}=$ 
$\left\{L_{1}^{i}, \ldots, L_{\gamma_{j}}^{i}\right\}$ with codim $L_{j}^{i}=l_{j}^{i}$. Then we define $N_{2, n}^{\mathrm{red}}\left(l_{0},\left\{l_{1}^{1}, \ldots, l_{\gamma_{1}}^{1}\right\},\left\{l_{1}^{2}, \ldots, l_{\gamma_{1}}^{2}\right\}\right)$ as the number of reducible conics $C_{1} \cup_{p} C_{2}$ in $\mathbb{C} P^{n}$ such that $C_{i}$ intersects all spaces in $\omega^{i}$ for $i=1,2$ and $p \in L_{0}$.

Lemma 2.9. With the hypothesis above, we have

$$
N_{2, n}^{\mathrm{red}}\left(l_{0},\left\{l_{1}^{1}, \ldots, l_{\gamma_{1}}^{1}\right\},\left\{l_{1}^{2}, \ldots, l_{\gamma_{2}}^{2}\right\}\right)=\prod_{i=1}^{2} N_{1, n}\left(2 n-1-\sum_{j=1}^{\gamma_{i}}\left(l_{j}^{i}-1\right), l_{1}^{i}, \ldots, l_{\gamma_{i}}^{i}\right) .
$$

Proof. Let $V_{i}$ be the algebraic variety in $\mathbb{C} P^{n}$ given by the union of all lines passing through all linear spaces in $\omega^{i}$. By definition, the number $N_{2, n}^{\mathrm{red}}\left(l_{0},\left\{l_{1}^{1}, \ldots, l_{\gamma_{1}}^{1}\right\}\right.$, $\left.\left\{l_{1}^{2}, \ldots, l_{\gamma_{1}}^{2}\right\}\right)$ is equal to the number of intersection points in $V_{1} \cap V_{2} \cap L_{0}$, i.e. is equal to the product of the degrees of $V_{1}$ and $V_{2}$. Since the configuration of linear spaces is generic, $V_{i}$ has dimension $2 n-1-\sum_{j=1}^{\gamma_{i}}\left(l_{j}^{i}-1\right)$. The degree of $V_{i}$ is the number of intersection points of $V_{i}$ with a generic linear space in $\mathbb{C} P^{n}$ of complementary dimension, and this number is by definition precisely $N_{1, n}\left(2 n-1-\sum_{j=1}^{\gamma_{i}}\left(l_{j}^{i}-1\right), l_{1}^{i}, \ldots, l_{\gamma_{i}}^{i}\right)$.

\section{Floor decomposition of tropical curves}

Here we explain how to enumerate complex curves of degree 1 and 2 with the help of the floor decomposition technique. This technique works for any degree (see [BM07], [BM08], [BM]) but the exposition of the method in its full generality would require a quite heavy formalism, which in our opinion would harm understanding this text. Hence we just give the main idea of the method before focusing on the degree 1 and 2 cases. Note that the floor decomposition technique has strong connections with the Caporaso and Harris method (see [CH98]), extended later by Vakil (see [Vak00b], [Vak00a]), and with the neck-stretching method in symplectic field theory (see [EGH00]).

We denote by $\pi: \mathbb{R}^{n} \rightarrow \mathbb{R}^{n-1}$ the linear projection forgetting the last coordinate. Given a minimal tropical morphism $f: C \rightarrow \mathbb{R}^{n}$, the morphism $\pi \circ f: C \rightarrow \mathbb{R}^{n-1}$ is not minimal in general. However, there exists a unique tropical curve $C^{\prime}$ equipped with a map $\rho: C \rightarrow C^{\prime}$ and a unique minimal tropical morphism $f^{\prime}: C^{\prime} \rightarrow \mathbb{R}^{n-1}$ such that $f=f^{\prime} \circ \rho$. We say that $f^{\prime}$ is induced by $\pi \circ f$, and that $f$ is a lifting of $f^{\prime}$.

\subsection{General method}

The starting idea of floor decomposition is to compute the numbers $N_{d, n}\left(l_{1}, \ldots, l_{\gamma}\right)$ by induction on the dimension $n$. As easy as it sounds, this approach does not work straightforwardly and one has to act carefully: It is easy to compute that through one point $p$ and two tropical lines $L_{1}$ and $L_{2}$ in $\mathbb{R}^{3}$ passes exactly one tropical line $L$ (see Example 4). However, there exist infinitely many tropical lines in $\mathbb{R}^{2}$ passing trough $\pi(p), \pi\left(L_{1}\right)$, and $\pi\left(L_{2}\right)$, and without knowing $L$, it is not clear at all which one of these planar lines is $\pi(L)$.

To make the induction work, we first stretch the configuration $\omega$ in the direction $U_{n}=$ $(0, \ldots, 0,-1)$. Then the tropical curves we are counting break into several floors for which we can apply induction. 
Example 4. Let us explain how to use the floor decomposition technique in a simple case. Let us choose a point $p$ and two tropical lines $L_{1}$ and $L_{2}$ in $\mathbb{R}^{3}$ such that $L_{1}$ (resp. $\left.L_{2}\right)$ consists of only one edge with direction $(0,1,0)$ (resp. $\left.(1,0,0)\right)$ contained in the horizontal plane with equation $z=a_{1}$ (resp. $z=a_{2}$ ). If the third coordinate of $p$ is much greater than $a_{1}$ which in its turn is much greater than $a_{2}$, then the unique tropical line $L$ in $\mathbb{R}^{3}$ passing through $p, L_{1}$, and $L_{2}$ is depicted in Figure 4 , and $\pi(L)$ is the unique tropical line in $\mathbb{R}^{2}$ passing through $\pi(p)$ and $\pi\left(L_{1}\right) \cap \pi\left(L_{2}\right)$.

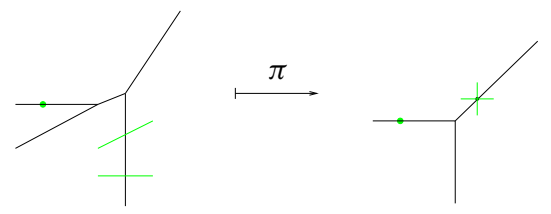

Fig. 4. Floor decomposition technique to compute $N_{1,3}(3,2,2)=1$.

Definition 3.1. Let $C$ be a rational tropical curve and $f: C \rightarrow \mathbb{R}^{n}$ a tropical morphism. An elevator of $f$ is an edge $e$ of $C$ with $u_{f, e}=(0, \ldots, 0, \pm 1)$. A floor of $f$ is a connected component of $C$ minus all its elevators.

Note that if $f$ is a morphism of degree $d$ in $\mathbb{R}^{n}$ and $\mathcal{F}$ is a floor of $f$, then $C$ induces a structure of tropical curve on $\mathcal{F}$ and $\pi \circ f_{\mid \mathcal{F}}: \mathcal{F} \rightarrow \mathbb{R}^{n-1}$ is a tropical morphism of degree $1 \leq d^{\prime} \leq d$. The integer $d^{\prime}$ is called the degree of $\mathcal{F}$.

Example 5. Examples of planar and spatial conics are depicted in Figure 5. Elevators are indicated by dotted lines.

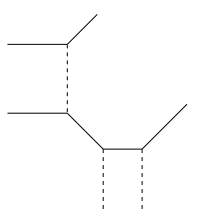

(a) A planar conic with two floor

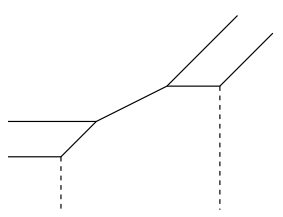

(b) A planar conic with one floor

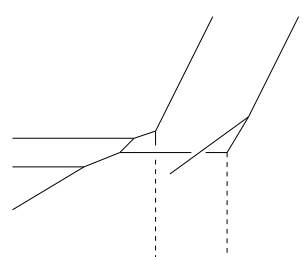

(c) A spatial conic with one floor

Fig. 5

Definition 3.1 extends to any tropical varieties in $\mathbb{R}^{n}$, but we keep restricting ourselves to complete tropical linear spaces. 
Definition 3.2. Let $L$ be a complete tropical linear space in $\mathbb{R}^{n}$ of codimension $j$. The wall (resp. floor) of $L$ is the union of all faces of $L$ of codimension $j$ which contain (resp. do not contain) the direction $(0, \ldots, 0,1)$.

Note that if $L$ is a complete tropical linear space of codimension $j$ in $\mathbb{R}^{n}$ with wall $W$ and floor $F$, then $\pi(W)$ is a complete tropical linear space of codimension $j$ in $\mathbb{R}^{n-1}$, and $\pi(F)=\pi(L)$ is a complete tropical linear space of codimension $j-1$ in $\mathbb{R}^{n-1}$.

Example 6. A tropical plane $L$ in $\mathbb{R}^{3}$ together with its wall $W$ are depicted in Figure 6. Clearly, $\pi(L)=\mathbb{R}^{2}$ and $\pi(W)$ is a tropical line in $\mathbb{R}^{2}$.

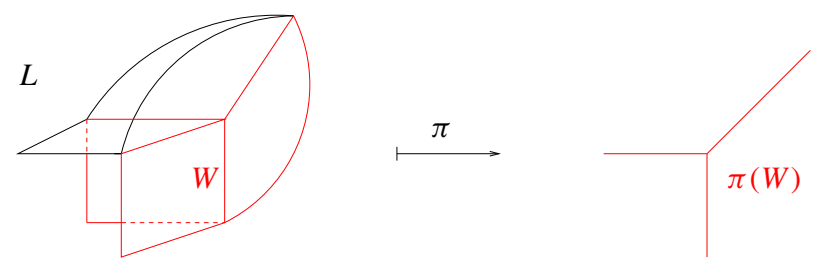

Fig. 6. $\pi(L)=\mathbb{R}^{2}$ and $\pi(W)$ is a tropical line.

Let us fix some integers $d \geq 1, n \geq 2, \gamma \geq 2, l_{1} \geq 1, \ldots, l_{\gamma} \geq 1$ subject to equality (1), and let us choose a generic configuration $\omega=\left\{L_{1}, \ldots, L_{\gamma}\right\}$ of complete tropical linear spaces in $\mathbb{R}^{n}$ such that codim $L_{j}=l_{j}$ for $j=1, \ldots, \gamma$. As before, $\mathbb{T} \mathcal{C}(\omega)$ is the set of all rational tropical morphisms $f: C \rightarrow \mathbb{R}^{n}$ of degree $d$ such that $f(C)$ intersects all tropical linear spaces in $\omega$.

Definition 3.3. An element $L$ of $\omega$ is called a vertical (resp. horizontal) constraint for $f \in \mathbb{T} \mathcal{C}(\omega)$ if $f(C) \cap L$ lies in the wall (resp. floor) of $L$.

Let us denote by $\operatorname{Vert}\left(L_{j}\right)$ the set of vertices of the complete tropical linear space $L_{j}$, and let us fix a hypercube $\mathcal{H}_{n-1}$ in $\mathbb{R}^{n-1}$ such that the cylinder $\mathcal{H}_{n-1} \times \mathbb{R}$ contains the set $\bigcup_{j=1}^{\gamma} \operatorname{Vert}\left(L_{j}\right)$. Given two points $v=\left(v_{1}, \ldots, v_{n}\right)$ and $w=\left(w_{1}, \ldots, w_{n}\right)$ in $\mathbb{R}^{n}$, we define $|v-w|_{n}=\left|v_{n}-w_{n}\right|$. Finally, we define $R_{\mathcal{H}}$ to be the length of the edges of $\mathcal{H}_{n-1}$, and

$$
R(\omega)=\min _{j \neq k, v \in \operatorname{Vert}\left(L_{j}\right), w \in \operatorname{Vert}\left(L_{k}\right)}|v-w|_{n} .
$$

The following observation is the key point of the technique.

Proposition 3.4 (Brugallé-Mikhalkin [BM07], [BM08], [BM]). There exists a real number $D(n, d)$, depending only on $n$ and $d$, such that if $R(\omega) \geq R_{\mathcal{H}} D(n, d)$ then for each morphism $f: C \rightarrow \mathbb{R}^{n}$ in $\mathbb{T} \mathcal{C}(\omega)$ and for each floor $\mathcal{F}$ of $f, f(\mathcal{F})$ meets exactly one horizontal constraint.

Definition 3.5. If $R(\omega) \geq R_{\mathcal{H}} D(n, d)$, we say that $\omega$ is a $(d, n)$-decomposing configuration. 
Note that requiring a configuration $\omega^{\mathbb{T}}$ to be $(d, n)$-decomposing is the same as imposing conditions on the relative position of the vertices of elements of $\omega^{\mathbb{T}}$. In particular, it makes sense to say that a configuration $\omega^{\mathbb{T}}$ is $(d, n)$-decomposing even if its elements do not satisfy equality (1).

The choice of the preferred direction $U_{n}$ provides a natural partial order among the tropical linear spaces.

Definition 3.6. Let $L$ and $L^{\prime}$ in $\mathbb{R}^{n}$ be two complete tropical linear spaces. We say that $L$ is higher than $L^{\prime}$, and write $L \gg L^{\prime}$, if any vertex of $L$ has last coordinate greater than all vertices of $L^{\prime}$.

Note that $\mathbb{R}^{n}$ is higher than any other tropical linear space. Before explaining in detail the case of lines and conics, we need to introduce a notation. Given a generic configuration $\left\{L_{1}, \ldots, L_{\gamma}\right\}$ of complete tropical linear spaces in $\mathbb{R}^{n}, k \in\{1, \ldots, \gamma\}$, and $A \subset\{1, \ldots, \gamma\}$, we define the following complete tropical linear spaces in $\mathbb{R}^{n-1}$ :

$$
L_{k}^{\prime}=\pi\left(W_{k}\right), \quad \widehat{L}_{k}^{\prime}=\pi\left(L_{k}\right), \quad \text { and } \quad \widetilde{L}_{A}^{\prime}=\bigcap_{j \in A} \pi\left(L_{j}\right),
$$

where $W_{j}$ is the wall of $L_{j}$. We also denote by $\widetilde{L}_{k}^{\prime}$ the complete tropical linear space $\widetilde{L}_{\{1, \ldots, k\}}^{\prime}$.

\subsection{The case $d=1$}

Suppose that $d=1$, so that $\sum_{j=1}^{\gamma}\left(l_{j}-1\right)=2 n-2$. We choose a $(1, n)$-decomposing configuration $\omega=\left\{L_{1}, \ldots, L_{\gamma}\right\}$ of complete tropical linear spaces in $\mathbb{R}^{n}, n \geq 3$, such that $L_{j}$ has codimension $l_{j}$ and $L_{j+1}$ is higher than $L_{j}$ for $j=1, \ldots, \gamma-1$. We denote by $W_{k}$ the wall of the constraint $L_{k}$. We denote by $\mathbb{T C}(\omega)^{(k)}$ the subset of tropical morphisms in $\mathbb{T C}(\omega)$ whose floor meets the horizontal constraint $L_{k}$ (remember that in degree one, the floor is unique). According to Proposition 3.4, we have

$$
\mathbb{T} \mathcal{C}(\omega)=\bigsqcup_{k=1}^{\gamma} \mathbb{T} \mathcal{C}(\omega)^{(k)}
$$

Given $k$ in $\{1, \ldots, \gamma\}$, we define $\omega^{\prime(k)}=\left\{\widetilde{L}_{k-1}^{\prime}, \widehat{L}_{k}^{\prime}, L_{k+1}^{\prime}, \ldots, L_{\gamma}^{\prime}\right\}$. Since $\omega$ is generic, the tropical linear space $L_{k}^{\prime}$ has codimension $l_{k}, \widehat{L}_{k}^{\prime}$ has codimension $l_{k}-1$, and $\widetilde{L}_{k-1}^{\prime}$ has codimension $\sum_{j=1}^{k-1}\left(l_{j}-1\right)$ if non-empty. If $f: C \rightarrow \mathbb{R}^{n}$ is an element of $\mathbb{T C}(\omega)^{(k)}$, then the tropical morphism $\pi \circ f: C \rightarrow \mathbb{R}^{n}$ induces obviously an element of $\mathbb{T} \mathcal{C}\left(\omega^{\prime(k)}\right)$. Moreover, any tropical morphism $f^{\prime}$ in $\mathbb{T} \mathcal{C}\left(\omega^{\prime(k)}\right)$ can be lifted in a unique way as an element $f$ of $\mathbb{T C}(\omega)^{(k)}$ : the only unknown is the location of the elevator of $f$, which is given by the unique intersection point of $f^{\prime}\left(C^{\prime}\right)$ with $\widetilde{L}_{k-1}^{\prime}$ (see Examples 4 and 7 ). Hence, there exists a natural bijection between the two sets $\mathbb{T} \mathcal{C}(\omega)^{(k)}$ and $\mathbb{T} \mathcal{C}\left(\omega^{\prime(k)}\right)$. Moreover this bijection respects multiplicity of tropical curves. In other words, we have the following proposition: 
Proposition 3.7 (Brugallé-Mikhalkin [BM07], [BM08], [BM]). For any $k$ in $\{1, \ldots, \gamma\}$, we have

$$
\sum_{f \in \mathbb{C} \mathcal{C}(\omega)^{(k)}} \mu(f)=\sum_{f^{\prime} \in \mathbb{T} \mathcal{C}\left(\omega^{\prime(k)}\right)} \mu\left(f^{\prime}\right) .
$$

Note that $\mathbb{T} \mathcal{C}\left(\omega^{\prime(k)}\right)=\emptyset$ if $k=1$ or $\sum_{j=1}^{k-1}\left(l_{j}-1\right)>n$. Proposition 3.7 allows one to compute all the numbers $N_{1, n}$ out of the numbers $N_{1, n-1}$. Since it is trivial that $N_{1,2}(2)=1$, all the numbers $N_{1, n}$ can be computed using Proposition 3.7.

Example 7. Let $\omega=\left\{L_{1}, L_{2}, L_{3}, L_{4}\right\}$ be a (1,3)-decomposing configuration of four lines in $\mathbb{R}^{3}$ with $L_{j+1} \gg L_{j}$. The set $\mathbb{T C}(\omega)^{(k)}$ is non-empty only for $k=2,3$, and the corresponding projected configurations in $\mathbb{R}^{2}$ are $\omega^{\prime(2)}=\left\{\pi\left(L_{1}\right), \pi\left(L_{2}\right), \pi\left(W_{3}\right), \pi\left(W_{4}\right)\right\}$ and $\omega^{\prime(3)}=\left\{\pi\left(L_{1}\right) \cap \pi\left(L_{2}\right), \pi\left(L_{3}\right), \pi\left(W_{4}\right)\right\}$ (see Figure 7a). Since there exists only one line passing through two points in the plane, we get $N_{1,3}(2,2,2,2)=1+1=2$.
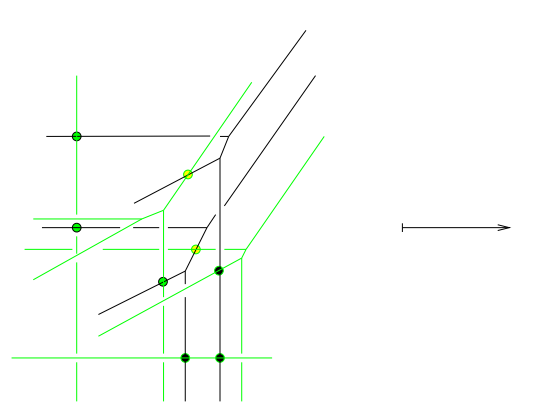

(a)

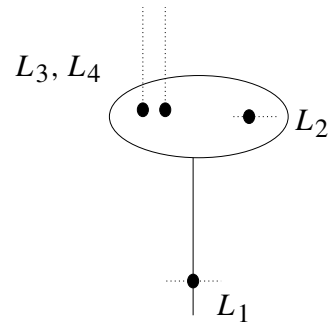

(b) Line in $\mathbb{T} \mathcal{C}(\omega)^{(2)}$
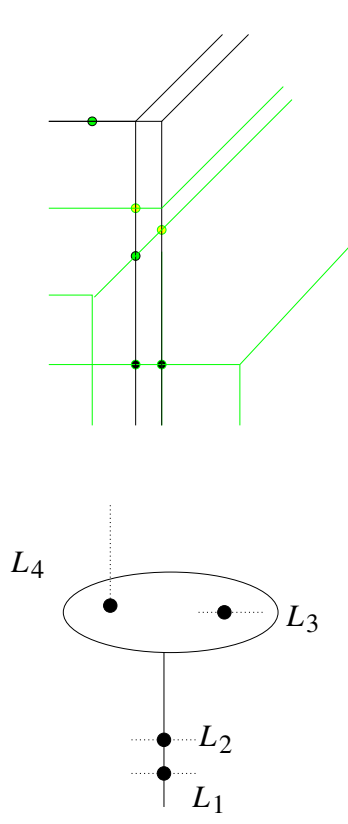

(c) Line in $\mathbb{T C}(\omega)^{(3)}$

Fig. 7. Floor decomposition technique to compute $N_{1,3}(2,2,2,2)=2$.

To depict tropical curves passing through a decomposing configuration, we use the following convention: a floor (resp. elevator) of the curve is represented by an ellipse (resp. a vertical edge); a constraint intersecting a floor (resp. elevator) is depicted by a dotted segment intersecting the corresponding ellipse (resp. vertical edge); a constraint is represented by a horizontal (resp. vertical) segment if it is a horizontal (resp. vertical) constraint for the curve. 
For example, the two tropical lines passing through the four lines $L_{1}, \ldots, L_{4}$ are represented by the diagrams depicted in Figures $7 \mathrm{~b}$ and $\mathrm{c}$.

Corollary 3.8. Given $l_{1}, \ldots, l_{\gamma} \geq 1$ such that $\sum_{j=1}^{\gamma}\left(l_{j}-1\right)=2 n-2$, we have

$$
N_{1, n}\left(l_{1}, \ldots, l_{\gamma}\right)=\sum_{k=2}^{\gamma} N_{1, n-1}\left(\sum_{i=1}^{k-1}\left(l_{i}-1\right), l_{k}-1, l_{k+1}, \ldots, l_{\gamma}\right) .
$$

Example 8. Let us compute the numbers $C(n, l)=N_{1, n}\left(l, l_{1}, \ldots, l_{2 n-1-l}\right)$ with $2 \leq$ $l \leq n$ and $l_{1}=\cdots=l_{2 n-1-l}=2$. According to Corollary 3.8, for any $n \geq 2$ and $3 \leq l \leq n$ we get

$$
C(n, l)=C(n-1, l-1)+C(n, l+1) .
$$

Hence we can extend the definition of the numbers $C(n, l)$ for all pairs $(n, l)$ with $n \geq 1$ according to relation (2), which is a Pascal type relation. The sequence

$$
A(n, l)=\left(\begin{array}{c}
2 n-l-1 \\
n-1
\end{array}\right)-\left(\begin{array}{c}
2 n-l-1 \\
n
\end{array}\right)
$$

also satisfies relation (2), and we have

$$
\forall n \geq 1 \quad C(n, 0)=A(n, 0)=0 \quad \text { and } \quad C(n, n)=A(n, n)=1
$$

so these two sequences must be equal on the set $\left\{(n, l) \in \mathbb{Z}^{2}: n \geq 1\right\}$. Hence we get

$$
\forall n \geq 2, \forall l \in\{2, \ldots, n\}, \quad C(n, l)=\left(\begin{array}{c}
2 n-l-1 \\
n-1
\end{array}\right)-\left(\begin{array}{c}
2 n-l-1 \\
n
\end{array}\right) .
$$

In particular, we find again the Catalan numbers

$$
C(n, 2)=C(n, 1)=\frac{1}{n}\left(\begin{array}{c}
2 n-2 \\
n-1
\end{array}\right)
$$

Note that Corollary 3.8 and (3) almost immediately imply that

$\forall n \geq 2, \forall k, l \in\{2, \ldots, n\}, N_{1, n}\left(k, l, l_{1}, \ldots, l_{2 n-k-l}\right)=\left(\begin{array}{c}2 n-l-k \\ n-k\end{array}\right)-\left(\begin{array}{c}2 n-l-k \\ n\end{array}\right)$ where $l_{1}=\cdots=l_{2 n-k-l}=2$.

\subsection{The case $d=2$}

Let us suppose that $d=2$, so that $\sum_{j=1}^{\gamma}\left(l_{j}-1\right)=3 n-1$. We choose a $(2, n)$ decomposing configuration $\omega=\left\{L_{1}, \ldots, L_{\gamma}\right\}$ of complete tropical linear spaces in $\mathbb{R}^{n}$, $n \geq 3$, such that $L_{j}$ has codimension $l_{j} \geq 2$ and $L_{j+1}$ is higher than $L_{j}$ for $j=$ $1, \ldots, \gamma-1$. As in Section 3.2, we denote by $W_{k}$ the wall of the constraint $L_{k}$. Given an element $f$ of $\mathbb{T C}(\omega)$, either $f$ has one floor of degree 2 , or it has two floors of degree 1 .

Let us first deal with the case of a conic with one floor of degree 2 . Let $k$ be an integer in $\{1, \ldots, \gamma\}$, and $A \sqcup B$ a partition of $\{1, \ldots, k-1\}$ into two sets. We denote by $\mathbb{T} \mathcal{C}(\omega)^{(k, A, B)}$ the set of all tropical morphisms in $\mathbb{T} \mathcal{C}(\omega)$ with one floor $\mathcal{F}$ of degree 2 such that 
- the floor $\mathcal{F}$ meets the horizontal constraint $L_{k}$;

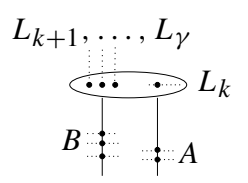

- one of the two elevators of $f$ meets all the constraints $L_{j}$ with $j \in A$, while the other elevator meets all the constraints $L_{j}$ with $j \in B$.

According to Proposition 3.4, the set of all tropical morphisms in $\mathbb{T C}(\omega)$ with a single floor is equal to

$$
\bigsqcup_{k=1}^{\gamma} \bigsqcup_{A \sqcup B=\{1, \ldots, k-1\}} \mathbb{T C}(\omega)^{(k, A, B)} .
$$

We define $\omega^{\prime(k, A, B)}=\left\{\widetilde{L}_{A}^{\prime}, \widetilde{L}_{B}^{\prime}, \widehat{L}_{k}^{\prime}, L_{k+1}^{\prime}, \ldots, L_{\gamma}^{\prime}\right\}$. The complete tropical linear space $L_{k}^{\prime}$ has codimension $l_{k}$, the space $\widehat{L}_{k}^{\prime}$ has codimension $l_{k}-1$, and $\widetilde{L}_{A}^{\prime}$ (resp. $\widetilde{L}_{B}^{\prime}$ ) has codimension $\sum_{j \in A}\left(l_{j}-1\right)$ (resp. $\left.\sum_{j \in B}\left(l_{j}-1\right)\right)$ if non-empty. As in Section 3.2, there is a natural map

$$
\phi_{(k, A, B)}: \mathbb{T} \mathcal{C}(\omega)^{(k, A, B)} \rightarrow \mathbb{T} \mathcal{C}\left(\omega^{\prime(k, A, B)}\right)
$$

In contrast to Section 3.2, the map $\phi_{(k, A, B)}$ is injective and respects multiplicity if and only if

$$
\operatorname{codim} \widetilde{L}_{A}^{\prime} \geq 2, \quad \operatorname{codim} \widetilde{L}_{B}^{\prime} \geq 2, \quad \text { and } \quad \operatorname{codim} \widehat{L}_{k}^{\prime} \geq 2
$$

In general, given $f^{\prime} \in \mathbb{T} C\left(\omega^{\prime(k, A, B)}\right)$ we have

$$
\sum_{f \in \phi_{(k, A, B)}^{-1}\left(f^{\prime}\right)} \mu(f)=2^{m_{(k, A, B)}} \mu\left(f^{\prime}\right)
$$

where $m_{(k, A, B)}$ is the number of spaces in $\left\{\widetilde{L}_{A}^{\prime}, \widetilde{L}_{B}^{\prime}, \widehat{L}_{k}^{\prime}\right\}$ of codimension 1 . The factor $2^{m_{(k, A, B)}}$ is just the manifestation of the fact that a conic in projective space intersects a hyperplane in two points (counted with multiplicity). Altogether we hence have the following proposition.

Proposition 3.9 (Brugallé-Mikhalkin [BM07], [BM08], [BM]). Given any $k, A$, and B as above, we have

$$
\sum_{f \in \mathbb{T} \mathcal{C}(\omega)^{(k, A, B)}} \mu(f)=2^{m_{(k, A, B)}} \sum_{f^{\prime} \in \mathbb{T} \mathcal{C}\left(\omega^{\prime(k, A, B)}\right)} \mu\left(f^{\prime}\right) .
$$

We now treat the case of tropical morphisms $f$ in $\mathbb{T C}(\omega)$ with two floors of degree 1 . Let $k_{1}<k_{2}$ be two integers in $\{1, \ldots, \gamma\}, A \sqcup B$ be a partition of $\left\{1, \ldots, k_{1}-1\right\}, D$ a subset of $\left\{k_{1}+1, \ldots, k_{2}-1\right\}$, and $C_{1} \sqcup C_{2}$ a partition of $\left\{k_{1}+1, \ldots, \gamma\right\} \backslash\left(\left\{k_{2}\right\} \cup D\right)$.

We denote by $\mathbb{T C}(\omega)^{\left(k_{1}, k_{2}, A, B, C_{1}, C_{2}, D\right)}$ the set of tropical morphisms in $\mathbb{T C}(\omega)$ with two floors $\mathcal{F}_{1}$ and $\mathcal{F}_{2}$ of degree 1 such that 
- the floor $\mathcal{F}_{i}$ meets the constraint $L_{k_{i}}$, and all the constraints $L_{j}$ with $j \in C_{i}$;

- one of the two unbounded elevators of $f$ meets all the constraints $L_{j}$ with $j \in A$, while the other unbounded elevator meets all the constraints $L_{j}$ with $j \in B$;

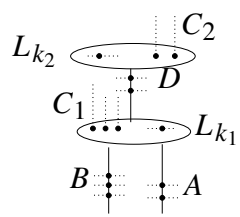

- the bounded elevator of $f$ meets all the constraints $L_{j}$ with $j \in D$.

According to Proposition 3.4, the set of all tropical morphisms in $\mathbb{T C}(\omega)$ with two floors is equal to

$$
\bigsqcup_{1 \leq k_{1}<k_{2} \leq \gamma} \bigsqcup_{A, B, C_{1}, C_{2}, D} \mathbb{T C}(\omega)^{\left(k_{1}, k_{2}, A, B, C_{1}, C_{2}, D\right)} .
$$

Given $i=1,2$, we define the following integers:

$$
\begin{aligned}
& l_{j}^{i}=l_{j} \text { if } j \in C_{i}, \quad l_{A}^{\prime}=\sum_{j \in A}\left(l_{j}-1\right), \quad l_{0}^{\prime 1}=2 n-\sum_{j \in C_{1}}\left(l_{j}^{\prime}-1\right)-l_{A}^{\prime}-l_{B}^{\prime}-l_{k_{1}}^{\prime}, \\
& l_{k_{i}}^{\prime}=l_{k_{i}}-1, \quad l_{B}^{\prime}=\sum_{j \in B}\left(l_{j}-1\right), \quad l_{0}^{\prime 2}=2 n-2-\sum_{j \in C_{2}}\left(l_{j}^{\prime}-1\right)-l_{k_{2}}^{\prime} .
\end{aligned}
$$

Finally, we denote by $\gamma_{i}$ the cardinals of the set $C_{i}$.

Proposition 3.10 (Brugallé-Mikhalkin [BM07], [BM08], [BM]). Given $k_{1}, k_{2}, A, B$, $C_{1}, C_{2}$, and $D$ as above, we have

$$
\begin{aligned}
\sum_{f \in \mathbb{T} C(\omega)^{\left(k_{1}, k_{2}, A, B, C_{1}, C_{2}, D\right)}} \mu(f) & \\
& =N_{1, n-1}\left(l_{i_{1}}^{\prime 1}, \ldots, l_{i_{\gamma_{1}}}^{\prime 1}, l_{A}^{\prime}, l_{B}^{\prime}, l_{k_{1}}^{\prime}, l_{0}^{\prime 1}\right) N_{1, n-1}\left(l_{j_{1}}^{\prime 2}, \ldots, l_{\gamma_{\gamma_{2}}}^{\prime 2}, l_{k_{2}}^{\prime}, l_{0}^{\prime 2}\right) .
\end{aligned}
$$

Let us give a heuristic of the proof of Proposition 3.10. We define $\omega^{1}=$ $\left\{\widetilde{L}_{A}^{\prime}, \widetilde{L}_{B}^{\prime}, \widehat{L}_{k_{1}}^{\prime}, L_{j}^{\prime}: j \in C_{1}\right\}, \omega^{\prime 2}=\left\{\widehat{L}_{k_{2}}^{\prime}, L_{j}^{\prime}: j \in C_{2}\right\}$, and $\omega^{\left(k_{1}, k_{2}, A, B, C_{1}, C_{2}, D\right)}=$ $\left(\widetilde{L}_{D}^{\prime}, \omega^{\prime 1}, \omega^{\prime 2}\right)$. As in Section 3.2, there is a natural and bijective map

$$
\phi: \mathbb{C} C(\omega)^{\left(k_{1}, k_{2}, A, B, C_{1}, C_{2}, D\right)} \rightarrow \mathbb{T} \mathcal{C}_{\mathrm{red}}\left(\omega^{\left(k_{1}, k_{2}, A, B, C_{1}, C_{2}, D\right)}\right)
$$

and Proposition 3.10 now follows from Lemma 2.9.

Once again, since it is trivial that $N_{2,1}(2)=1$ and $N_{2,2}(5)=1$, all the numbers $N_{n, 2}$ can be computed inductively using Propositions 3.7, 3.9, and 3.10.

Example 9 (see [BM07]). In Figure 8, we depict all possible floor decompositions for tropical conics in $\mathbb{R}^{3}$ passing through a $(2,3)$-decomposing configuration of eight tropical lines. In each case, we specify the number of $\left(k_{1}, k_{2}, A, B, C_{1}, C_{2}, D\right)$ or $(k, A, B)$ with a non-empty corresponding set of tropical morphisms, and the sum of the multiplicities of the corresponding curves in $\mathbb{T C}(\omega)^{\left(k_{1}, k_{2}, A, B, C_{1}, C_{2}, D\right)}$ or $\mathbb{T} \mathcal{C}(\omega)^{(k, A, B)}$ for each such choice. 

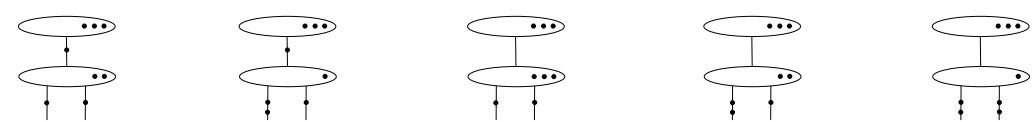

5, $\mu=1$

$3, \mu=1$

$10, \mu=1$

$12, \mu=1$

$3, \mu=1$
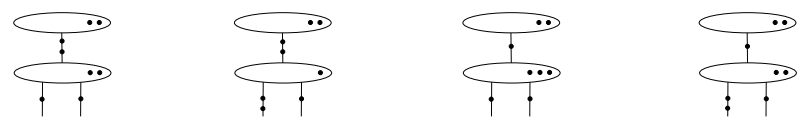

5, $\mu=1$

$3, \mu=1$

$10, \mu=1$

$12, \mu=1$
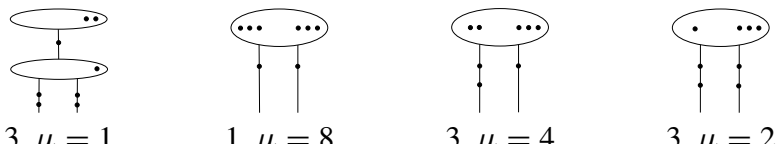

Fig. 8. Floor decompositions of the 92 tropical conics passing through eight lines in $\mathbb{R}^{3}$.

\section{Maximal configurations for conics}

\subsection{Well-ordered totally decomposing configurations}

To prove Theorem 1.1, we exhibit maximal configurations, that is, configurations $\omega$ of complete tropical linear spaces such that the cardinality $|\mathbb{T C}(\omega)|$ is equal to the corresponding Gromov-Witten invariant.

In this section, we define well-ordered totally decomposing configurations. The rest of the paper will be devoted to the proof that any such configuration is maximal when dealing with conics.

Definition 4.1. Let $\omega=\left\{L_{1}, \ldots, L_{\gamma}\right\}$ be a $(d, n)$-decomposing configuration of complete tropical linear spaces in $\mathbb{R}^{n}$, and let $W_{i}$ be the wall of $L_{i}$. We say that $\omega$ is a $(d, n)$ totally decomposing configuration if it satisfies one of the following two conditions:

- $n=2$;

- for any subset $\Gamma \subset\{1, \ldots, \gamma\}$ the configuration $\left\{\pi\left(L_{i}\right), \pi\left(W_{j}\right): i \in \Gamma, j \notin \Gamma\right\}$ is $(d, n-1)$-totally decomposing.

Since a hyperplane in $\mathbb{R}^{n}$ has a single vertex, the existence of totally decomposing configurations of tropical hyperplanes is straightforward. Now suppose that we want to construct a totally decomposing configuration $\omega=\left\{L_{1}, \ldots, L_{\gamma}\right\}$ with $\operatorname{codim} L_{i}=l_{i}$. We start with a totally decomposing configuration $\left\{H_{1}, \ldots, H_{\gamma}\right\}$ of tropical hyperplanes, and we construct $L_{i}$ by intersecting $l_{i}$ copies of $H_{i}$ translated by very small vectors.

Remark. Let $\left\{L_{1}, \ldots, L_{\gamma}\right\}$ be a generic totally decomposing configuration of complete tropical linear spaces, $\Gamma \subset\{1, \ldots, \gamma\}$, and $\omega^{\prime}$ the configuration $\left\{\pi\left(L_{i}\right), \pi\left(W_{j}\right): i \in \Gamma\right.$, $j \notin \Gamma$. Then it follows directly from Definition 4 .1 that given any elements $\mathcal{L}_{1}, \ldots, \mathcal{L}_{k}$ of $\omega^{\prime}$, the floor of the complete tropical linear space $\bigcap_{i=1}^{k} \mathcal{L}_{k}$ is contained in the floor of the lowest space among $\mathcal{L}_{1}, \ldots, \mathcal{L}_{k}$ (see Figure 9). 


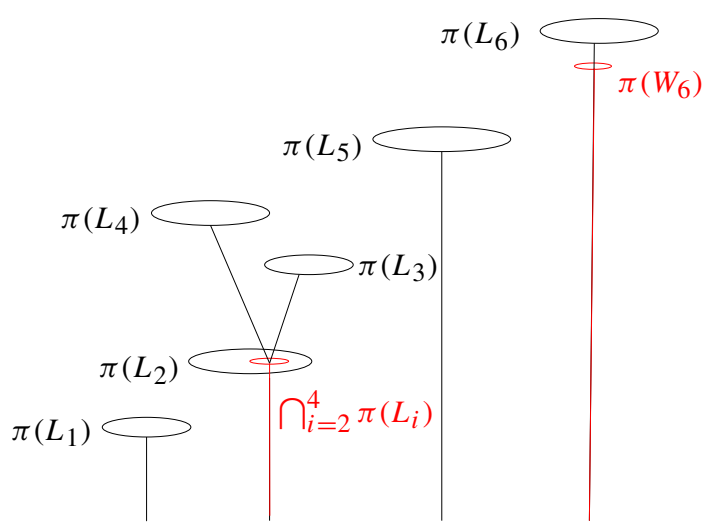

Fig. 9. $\omega=\left\{L_{1}, \ldots, L_{6}\right\}$.

The next lemma provides an alternative proof of Sottile's Theorem about maximality of real enumerative problems concerning lines in projective spaces.

Lemma 4.2. Let $\omega$ be a $(1, n)$-totally decomposing configuration of complete tropical linear spaces in $\mathbb{R}^{n}$ subject to equality (1) with $d=1$. Then $\omega$ is maximal.

Proof. We use induction on $n$. Clearly, the lemma is true for $n=2$. Suppose now that $n \geq 3$ and that the lemma is true in dimension $n-1$. In what follows, we use the notation of Section 3.2. Any projected configuration $\omega^{\prime(k)}$ in $\mathbb{R}^{n-1}$ is $(1, n-1)$-totally decomposing, and thus maximal by the induction hypothesis. Hence any tropical morphism $f$ in $\mathbb{T} \mathcal{C}\left(\omega^{\prime(k)}\right)$ has multiplicity 1 . Since the two sets $\mathbb{T} \mathcal{C}(\omega)^{(k)}$ and $\mathbb{T} \mathcal{C}\left(\omega^{\prime(k)}\right)$ have the same cardinality, we deduce from Proposition 3.7 that

$$
\sum_{f \in \mathbb{T} \mathcal{C}(\omega)} \mu(f)=\sum_{k=2}^{\gamma}\left|\mathbb{T} \mathcal{C}(\omega)^{(k)}\right|=|\mathbb{T} \mathcal{C}(\omega)| .
$$

In other words, the configuration $\omega$ is maximal.

It turns out that $(2, n)$-totally decomposing configurations are not necessarily maximal.

Definition 4.3. Let $\omega=\left\{L_{1}, \ldots, L_{\gamma}\right\}$ be a $(d, n)$-totally decomposing configuration of complete tropical linear spaces in $\mathbb{R}^{n}$, and let $W_{i}$ be the wall of $L_{i}$. We say that $\omega$ is a well-ordered $(d, n)$-totally decomposing configuration if it satisfies one of the following two conditions:

- $n=2$;

- for any subset $\Gamma \subset\{1, \ldots, \gamma\}$ the configuration $\left\{\pi\left(L_{i}\right), \pi\left(W_{j}\right): i \in \Gamma, j \notin \Gamma\right\}$ is a well-ordered $(d, n-1)$-totally decomposing configuration; moreover for any $i$ and $j$ such that $L_{i} \gg L_{j}$, we have $\pi\left(L_{i}\right) \gg \pi\left(L_{j}\right)$ and $\pi\left(W_{i}\right) \gg \pi\left(L_{j}\right)$ whenever the following three conditions hold: $\operatorname{codim} \pi\left(L_{j}\right) \geq 1, \pi\left(L_{i}\right) \gg \pi\left(W_{j}\right)$ and $\pi\left(W_{i}\right)$ $\gg \pi\left(W_{j}\right)$. 
Again it is trivial that well-ordered totally decomposing configurations of hyperplanes exist, from which it follows that there exists a well-ordered totally decomposing configuration $\omega=\left\{L_{1}, \ldots, L_{\gamma}\right\}$ with codim $L_{i}=l_{i}$ for any fixed positive integers $l_{1}, \ldots, l_{\gamma}$.

Remark. Let $\left\{L_{1}, \ldots, L_{\gamma}\right\}$ be a generic well-ordered totally decomposing configuration of complete tropical linear spaces, $\Gamma \subset\{1, \ldots, \gamma\}$, and $\omega^{\prime}$ the configuration $\left\{\pi\left(L_{i}\right), \pi\left(W_{j}\right): i \in \Gamma, j \notin \Gamma\right\}$. Then it follows directly from Definition 4.3 that given any elements $\mathcal{L}_{1}, \ldots, \mathcal{L}_{k}$ of $\omega^{\prime}$, the configuration obtained from $\omega^{\prime}$ by replacing $\mathcal{L}_{1}, \ldots, \mathcal{L}_{k}$ by $\bigcap_{i=1}^{k} \mathcal{L}_{i}$ is still a well-ordered totally decomposing configuration (see Figure 9, where $L_{6} \gg \cdots \gg L_{1}$ ).

We will prove in Proposition 4.5 that a well-ordered $(2, n)$-totally decomposing configuration is maximal. We will treat the case of tropical conics with two floors of degree one using the total decomposition hypothesis more or less as in Lemma 4.2 (see Proposition 4.7), and we will treat the case of tropical conics with one floor of degree 2 using the well-order hypothesis.

Before proving Proposition 4.5 in its full generality, let us first illustrate by a simple example how the well-order hypothesis solves the maximality problem for the case of tropical conics with one floor of degree 2.

\subsection{Conics through eight lines in $\mathbb{R}^{3}$}

Maximality of the real enumerative problem concerning conics passing through eight spatial lines in general position in $\mathbb{R} P^{3}$ was first announced by Brugallé and Mikhalkin in [BM07].

Let us fix a well-ordered (2, 3)-totally decomposing configuration $\omega=\left\{L_{1}, \ldots, L_{8}\right\}$ of eight tropical lines in $\mathbb{R}^{3}$. We suppose that $L_{8} \gg L_{7} \gg \cdots \gg L_{1}$, and we denote by $W_{i}$ the wall of $L_{i}$. Recall that notations have been defined in Section 3.3.

Lemma 4.4. If the triple $(k, A, B)$ is such that $\mathbb{T C}(\omega)^{(k, A, B)}$ is non-empty, then it contains exactly $2^{m_{(k, A, B)}}$ tropical morphisms, all of them of multiplicity 1.

Proof. Let us fix such a triple $(k, A, B)$. It is easy to see that the three higher lines $L_{6}, L_{7}$ and $L_{8}$ are vertical constraints for elements of $\mathbb{T} \mathcal{C}(\omega)^{(k, A, B)}$ (see Figure 8). In particular, the configuration $\omega^{\prime(k, A, B)}$ in $\mathbb{R}^{2}$ contains the three points $w_{8}=\pi\left(W_{8}\right), w_{7}=\pi\left(W_{7}\right)$, and $w_{6}=\pi\left(W_{6}\right)$. Since $\omega$ is a well-ordered $(2,3)$-totally decomposing configuration, the configuration $\omega^{\prime(k, A, B)}$ is $(2,2)$-decomposing and the points $w_{8}, w_{7}$, and $w_{6}$ are its highest elements. Moreover, $N_{2,2}(2,2,2,2,2)=1$ so $\omega^{\prime(k, A, B)}$ is maximal. Hence the set $\mathbb{T} \mathcal{C}\left(\omega^{\prime(k, A, B)}\right)$ reduces to a unique plane tropical conic $f: C \rightarrow \mathbb{R}^{2}$ (passing through the three points $w_{8}, w_{7}, w_{6}$, and two others) which intersects the $m_{(k, A, B)}=1,2$ or 3 tropical lines in $\omega^{\prime(k, A, B)}$.

It remains to show that each of these $m_{(k, A, B)}$ tropical lines intersects the tropical conic $f(C)$ in two distinct points, which would imply the lemma by Proposition 3.9. According to what we said above about the configuration $\omega^{\prime(k, A, B)}$, the tropical conic $f$ has two floors of degree 1 passing through the points $w_{8}$ and $w_{6}$, while the bounded 


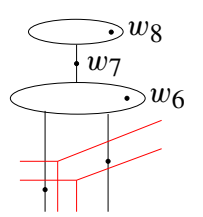

Fig. 10. Floor decomposition of a planar conic.

elevator of $f$ passes through $w_{7}$ (see Figure 10). Since any tropical line in $\omega^{\prime(k, A, B)}$ is lower than $w_{6}$, it must intersect $f(C)$ along its unbounded elevators. In particular, it intersects $f(C)$ in two distinct points.

It is immediate that the set $\mathbb{T C}(\omega)^{\left(k_{1}, k_{2}, A, B, C_{1}, C_{2}, D\right)}$ is either empty of composed of a unique tropical morphism of multiplicity 1 (see Figure 8 ). So the set $\mathbb{T C}(\omega)$ is composed of tropical morphisms of multiplicity 1 . Hence according to Lemma 2.6, there exists a generic configuration of eight lines in $\mathbb{R} P^{3}$ such that exactly 92 real conics intersect these eight lines.

\subsection{General case}

Theorem 1.1 is a direct consequence of Proposition 4.5, Theorem 2.5, and Lemma 2.6. The next proposition relies on Proposition 4.7, which is proved in the next section.

Proposition 4.5. Let $\omega$ be a well-ordered $(2, n)$-totally decomposing configuration of complete tropical linear spaces in $\mathbb{R}^{n}$ subject to equality (1). Then $\omega$ is maximal.

Proof. Our goal is to prove that $\sum_{f \in \mathbb{C} C(\omega)} \mu(f)=|\mathbb{T C}(\omega)|$. This is obviously true when $n=2$ since the left hand side is 1 . Suppose that $n \geq 3$ and the equality is true in lower dimensions. Since $\omega$ is $(2, n)$-decomposing, we have

$$
\begin{aligned}
\sum_{f \in \mathbb{T C}(\omega)} \mu(f) & \\
= & \sum_{(k, A, B)}\left(\sum_{f \in \mathbb{C} \mathcal{C}(\omega)^{(k, A, B)}} \mu(f)\right)+\sum_{\left(k_{1}, k_{2}, A, B, C_{1}, C_{2}, D\right)}\left(\sum_{f \in \mathbb{C} \mathcal{C}(\omega)^{\left(k_{1}, k_{2}, A, B, C_{1}, C_{2}, D\right)}} \mu(f)\right)
\end{aligned}
$$

where the sums are taken following Section 3.3.

Suppose that $\mathbb{T} \mathcal{C}(\omega)^{(k, A, B)}$ is non-empty. By induction, $\omega^{\prime(k, A, B)}$ is a well-ordered $(2, n-1)$-totally decomposing configuration and so is maximal. Moreover, the map $\phi_{(k, A, B)}: \mathbb{T} \mathcal{C}(\omega)^{(k, A, B)} \rightarrow \mathbb{T C}\left(\omega^{\prime(k, A, B)}\right)$ is $2^{m(k, A, B)}$-to-1, counted with multiplicities. Hence, it remains to show that any conic in $\mathbb{T} C\left(\omega^{\prime(k, A, B)}\right)$ intersects each of the $m(k, A, B)$ hyperplanes of $\omega^{\prime(k, A, B)}$ in two distinct points, which would imply, by Proposition 3.9, that

$$
\begin{aligned}
& \left|\mathbb{T} \mathcal{C}(\omega)^{(k, A, B)}\right|=2^{m(k, A, B)}\left|\mathbb{T} \mathcal{C}\left(\omega^{\prime(k, A, B)}\right)\right|=2^{m(k, A, B)} \sum_{f^{\prime} \in \mathbb{T} \mathcal{C}\left(\omega^{\prime(k, A, B)}\right)} \mu\left(f^{\prime}\right) \\
& =\sum_{f \in \mathbb{T} \mathcal{C}(\omega)^{(k, A, B)}} \mu(f) .
\end{aligned}
$$


We have $\omega^{\prime(k, A, B)}=\left\{\widetilde{L}_{A}^{\prime}, \widetilde{L}_{B}^{\prime}, \widehat{L}_{k}^{\prime}, L_{k+1}^{\prime}, \ldots, L_{\gamma}^{\prime}\right\}$, so a tropical hyperplane $H$ in $\omega^{\prime(k, A, B)}$ is either $\widehat{L}_{k}^{\prime}, \widetilde{L}_{A}^{\prime}$ or $\widetilde{L}_{B}^{\prime}$. Since $\omega^{\prime(k, A, B)}$ is a well-ordered configuration, $\widehat{L}_{k}^{\prime}, \widetilde{L}_{A}^{\prime}$ and $\widetilde{L}_{B}^{\prime}$ are its three lowest elements. In particular, $H$ is higher than at most two other elements of $\omega^{\prime(k, A, B)}$. The configuration $\omega^{\prime(k, A, B)}$ is $(2, n-1)$-decomposing, so any unbounded elevator and any floor of a conic in $\mathbb{T} C\left(\omega^{\prime(k, A, B)}\right)$ has to intersect at least one element of $\omega^{\prime(k, A, B)}$ which is not a hyperplane. In particular, a hyperplane in $\omega^{\prime(k, A, B)}$ intersects such a conic strictly below the lowest floor, that is, along its two unbounded elevators in two distinct points (see Figure 11).

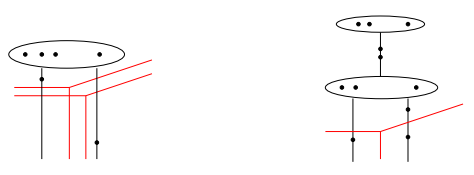

Fig. 11. Floor decompositions in $\mathbb{T C}\left(\omega^{\prime(k, A, B)}\right)$ and hyperplanes.

Suppose that $\mathbb{T C}(\omega)^{\left(k_{1}, k_{2}, A, B, C_{1}, C_{2}, D\right)}$ is non-empty. According to Section 3.3, we have

$$
\begin{aligned}
\left|\mathbb{T} \mathcal{C}_{\text {red }}\left(\omega^{\left(k_{1}, k_{2}, A, B, C_{1}, C_{2}, D\right)}\right)\right|=\left|\mathbb{T C}(\omega)^{\left(k_{1}, k_{2}, A, B, C_{1}, C_{2}, D\right)}\right| \\
\leq \sum_{f \in \mathbb{T} \mathcal{C}(\omega)^{\left(k_{1}, k_{2}, A, B, C_{1}, C_{2}, D\right)}} \mu(f) \\
\leq N_{1, n-1}\left(l_{i_{1}}^{\prime 1}, \ldots, l_{i_{\gamma_{1}}}^{\prime 1}, l_{A}^{\prime}, l_{B}^{\prime}, l_{k_{1}}^{\prime}, l_{0}^{\prime 1}\right) N_{1, n-1}\left(l_{j_{1}}^{\prime 2}, \ldots, l_{j_{\gamma_{2}}}^{\prime 2}, l_{k_{2}}^{\prime}, l_{0}^{\prime 2}\right) .
\end{aligned}
$$

According to Proposition 4.7 (see the next section) applied to $\omega^{\left(k_{1}, k_{2}, A, B, C_{1}, C_{2}, D\right)}=$ $\left(\widetilde{L}_{D}^{\prime}, \omega^{\prime 1}, \omega^{\prime 2}\right)$, we get

$$
\begin{aligned}
& \left|\mathbb{T} \mathcal{C}_{\text {red }}\left(\omega^{\left(k_{1}, k_{2}, A, B, C_{1}, C_{2}, D\right)}\right)\right| \\
& =N_{1, n-1}\left(l_{i_{1}}^{\prime 1}, \ldots, l_{i_{\gamma_{1}}}^{\prime 1}, l_{A}^{\prime}, l_{B}^{\prime}, l_{k_{1}}^{\prime}, l_{0}^{\prime 1}\right) N_{1, n-1}\left(l_{j_{1}}^{\prime 2}, \ldots, l_{\gamma_{\gamma_{2}}}^{\prime 2}, l_{k_{2}}^{\prime}, l_{0}^{\prime 2}\right) .
\end{aligned}
$$

Hence all inequalities in (4) are in fact equalities, and we have

$$
\left|\mathbb{T C}(\omega)^{\left(k_{1}, k_{2}, A, B, C_{1}, C_{2}, D\right)}\right|=\sum_{f \in \mathbb{C}(\omega)^{\left(k_{1}, k_{2}, A, B, C_{1}, C_{2}, D\right)}} \mu(f),
$$

which completes the proof of the proposition.

\subsection{Reducible conics through a well-ordered totally decomposing configuration}

A reducible tropical morphism $f: C \rightarrow \mathbb{R}^{n}$ of degree 2 has either two floors of degree 1 , or a unique (reducible) floor of degree 2. The proof of Proposition 3.4 only relies on the finiteness of the set $\mathbb{T C}(\omega)$ and on vectors $u_{f, e}$ for $e \in \operatorname{Edge}^{\infty}(C)$. In particular Lemma 2.8 implies that Proposition 3.4 still holds for elements of $\mathbb{T} \mathcal{C}_{\text {red }}\left(L_{0}, \omega^{1}, \omega^{2}\right)$. In this section we compute the numbers of reducible tropical morphisms of degree 2 passing through a particular configuration of complete tropical linear spaces in $\mathbb{R}^{n}$. 
Let $l_{0} \geq 0$ and $l_{1}^{1}, \ldots, l_{\gamma_{1}}^{1}, l_{1}^{2}, \ldots, l_{\gamma_{2}}^{2} \geq 1$ be some integers such that

$$
l_{0}+\sum_{i=1}^{2} \sum_{j=1}^{\gamma_{i}}\left(l_{j}^{i}-1\right)=3 n-2
$$

We choose a complete tropical linear space $L_{0}$ in $\mathbb{R}^{n}$ of codimension $l_{0}$ and two configurations $\omega^{1}$ and $\omega^{2}$ of complete tropical linear spaces in $\mathbb{R}^{n}$ such that $\omega^{i}=\left\{L_{1}^{i}, \ldots, L_{\gamma_{i}}^{i}\right\}$ with $\operatorname{codim} L_{j}^{i}=l_{j}^{i}$ and $L_{j}^{i} \gg L_{j+1}^{i}$.

Definition 4.6. The configuration $\left\{L_{0}, \omega^{1}, \omega^{2}\right\}$ is said to be separated if the configuration $\left\{L_{0}\right\} \cup \omega^{1} \cup \omega^{2}$ is a well-ordered $(2, n)$-totally decomposing configuration, and if $L_{0}$ and elements of $\omega^{1}$ are below elements of $\omega^{2}$.

Note that we do not make any assumption about the mutual position of $L_{0}$ and elements of $\omega^{1}$, and that the projection to $\mathbb{R}^{n-1}$ of a separated configuration in $\mathbb{R}^{n}$ is still separated. Given a separated configuration $\left\{L_{0}, \omega^{1}, \omega^{2}\right\}$ in $\mathbb{R}^{n}$, we denote by $\mathbb{T} N_{n}^{\text {red }}\left(l_{0},\left\{l_{1}^{1}, \ldots, l_{\gamma_{1}}^{1}\right\},\left\{l_{1}^{2}, \ldots, l_{\gamma_{2}}^{2}\right\}\right)$ the cardinality of $\mathbb{T} \mathcal{C}_{\text {red }}\left(L_{0}, \omega^{1}, \omega^{2}\right)$. We will see in Proposition 4.7 that this cardinality does not depend on $L_{0}, \omega^{1}$, and $\omega^{2}$ as long as $\left\{L_{0}, \omega^{1}, \omega^{2}\right\}$ is separated.

Proposition 4.7. For any generic separated configuration $\left\{L_{0}, \omega^{1}, \omega^{2}\right\}$ in $\mathbb{R}^{n}$, we have

$$
\mathbb{T} N_{n}^{\mathrm{red}}\left(l_{0},\left\{l_{1}^{1}, \ldots, l_{\gamma_{1}}^{1}\right\},\left\{l_{1}^{2}, \ldots, l_{\gamma_{2}}^{2}\right\}\right)=\prod_{i=1,2} N_{1, n}\left(2 n-1-\sum_{j=1}^{\gamma_{i}}\left(l_{j}^{i}-1\right), l_{1}^{i}, \ldots, l_{\gamma_{i}}^{i}\right) .
$$

Proof. The case $n=2$ is straightforward. Suppose now that $n \geq 3$ and that the proposition is true in lower dimensions.

Let $f: C_{1} \cup_{p} C_{2} \rightarrow \mathbb{R}^{n}$ be an element of $\mathbb{T} \mathcal{C}_{\text {red }}\left(L_{0}, \omega^{1}, \omega^{2}\right)$. An elevator of $f$ has to meet at least one constraint, and the elements of $\omega^{2}$ are above $L_{0}$ and the elements of $\omega^{1}$, so $f$ has two floors of degree 1 ; moreover the node of $C$ is either on both elevators of $C_{1}$ and $C_{2}$, or on the floor of $C_{1}$ and the elevator of $C_{2}$ (see Figure 12).
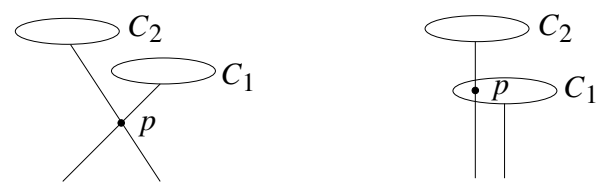

Fig. 12

We define $k_{0}$ as the smallest integer such that $L_{k_{0}}^{1}$ is higher than $L_{0}$ if such an element of $\omega^{1}$ exists, and by $k_{0}=0$ otherwise. We denote by $\mathcal{F}_{i}$ the floor of $C_{i}, i=1,2$.

We consider the partition

$$
\bigsqcup_{\substack{k_{0} \leq k_{1} \leq \gamma_{1} \\ 1 \leq k_{2} \leq \gamma_{2}}} \mathcal{C}_{1}^{k_{1}, k_{2}} \sqcup \bigsqcup_{1 \leq k_{2} \leq \gamma_{2}} \mathcal{C}_{2}^{k_{2}} \sqcup \bigsqcup_{\substack{1 \leq k_{1} \leq k_{0}-1 \\ 1 \leq k_{2} \leq \gamma_{2}}} \mathcal{C}_{3}^{k_{1}, k_{2}}
$$

of the set $\mathbb{T} \mathcal{C}_{\text {red }}\left(L_{0}, \omega^{1}, \omega^{2}\right)$ where 
- $\mathcal{C}_{1}^{k_{1}, k_{2}}$ is the set of all elements $f_{1} \cup_{p} f_{2}: C_{1} \cup_{p} C_{2} \rightarrow \mathbb{R}^{n}$ in $\mathbb{T} \mathcal{C}_{\text {red }}\left(L_{0}, \omega^{1}, \omega^{2}\right)$ such that $p$ is on both elevators of $f_{1}$ and $f_{2}$, and the floor $\mathcal{F}_{i}$ meets the horizontal constraint $L_{k_{i}}^{i}$; note that $\mathcal{C}_{1}^{k_{1}, k_{2}}=\emptyset$ if $k_{0}=0$;

- $\mathcal{C}_{2}^{k_{2}}$ is the set of all elements $f_{1} \cup_{p} f_{2}: C_{1} \cup_{p} C_{2} \rightarrow \mathbb{R}^{n}$ in $\mathbb{T} \mathcal{C}_{\text {red }}\left(L_{0}, \omega^{1}, \omega^{2}\right)$ such that $p$ is on the floor $\mathcal{F}_{1}, L_{0}$ is the horizontal constraint of $\mathcal{F}_{1}$, and the floor $\mathcal{F}_{2}$ meets the horizontal constraint $L_{k_{2}}^{2}$; note that $\mathcal{C}_{2}^{k_{2}}=\emptyset$ if $k_{0} \leq 1$;

- $\mathcal{C}_{3}^{k_{1}, k_{2}}$ is the set of all elements $f_{1} \cup_{p} f_{2}: C_{1} \cup_{p} C_{2} \rightarrow \mathbb{R}^{n}$ in $\mathbb{T} \mathcal{C}_{\text {red }}\left(L_{0}, \omega^{1}, \omega^{2}\right)$ such that $p$ is on the floor $\mathcal{F}_{1}$, and the floor $\mathcal{F}_{i}$ meets the horizontal constraint $L_{k_{i}}^{i}$.

We denote by $W_{j}^{i}$ (resp. $W_{0}$ ) the wall of the constraint $L_{j}^{i}$ (resp. $L_{0}$ ). We consider the following complete tropical linear spaces in $\mathbb{R}^{n-1}$ :

$$
\begin{gathered}
L_{j}^{\prime i}=\pi\left(W_{j}^{i}\right), \quad \widehat{L}_{j}^{\prime i}=\pi\left(L_{j}^{i}\right), \quad \widetilde{L}_{0}^{\prime k_{1}, k_{2}}=\pi\left(L_{0}\right) \cap \bigcap_{i=1,2} \bigcap_{1 \leq j \leq k_{i}-1} \pi\left(L_{j}^{i}\right), \\
\widetilde{L}_{k}^{\prime 1}=\bigcap_{1 \leq j \leq k-1} \pi\left(L_{j}^{1}\right), \quad \widetilde{L}_{0}^{\prime k_{2}}=\pi\left(L_{0}\right) \cap \bigcap_{1 \leq j \leq k_{2}-1} \pi\left(L_{j}^{2}\right), \\
\mathcal{L}_{0}^{\prime k_{2}}=\pi\left(W_{0}\right) \cap \bigcap_{1 \leq j \leq k_{2}-1} \pi\left(L_{j}^{2}\right),
\end{gathered}
$$

and the following configurations:

$$
\begin{aligned}
\omega_{1}^{i, k_{i}} & =\left\{\widehat{L}_{k_{i}}^{\prime i}, L_{k_{i}+1}^{\prime i}, \ldots, L_{\gamma_{i}}^{\prime i}\right\} \quad \text { for } i=1,2, \\
\omega_{2}^{1} & =\left\{\widetilde{L}_{k_{0}}^{\prime 1}, L_{k_{0}}^{\prime 1}, \ldots, L_{\gamma_{1}}^{\prime 1}\right\}, \quad \omega_{3}^{1, k_{1}}=\left\{\widehat{L}_{k_{1}}^{\prime 1}, \widetilde{L}_{k_{1}}^{\prime 1}, L_{k_{1}+1}^{\prime 1}, \ldots, L_{\gamma_{1}}^{\prime 1}\right\} .
\end{aligned}
$$

Given an element $f$ of $\mathcal{C}_{1}^{k_{1}, k_{2}}$, the tropical morphism $\pi \circ f$ induces an element of $\mathbb{T} \mathcal{C}_{\text {red }}\left(\widetilde{L}_{0}^{\prime k_{1}, k_{2}}, \omega_{1}^{1, k_{1}}, \omega_{1}^{2, k_{2}}\right)$. Conversely any element $f_{1}^{\prime} \cup_{p^{\prime}} f_{2}^{\prime}: C_{1}^{\prime} \cup_{p^{\prime}} C_{2}^{\prime} \rightarrow \mathbb{R}^{n-1}$ of $\mathbb{T} \mathcal{C}_{\text {red }}\left(\widetilde{L}_{0}^{\prime k_{1}, k_{2}}, \omega_{1}^{1, k_{1}}, \omega_{1}^{2, k_{2}}\right)$ has a unique lift $f_{1} \cup_{p} f_{2}: C_{1} \cup_{p} C_{2} \rightarrow \mathbb{R}^{n}$ in $\mathcal{C}_{1}^{k_{1}, k_{2}}$; the elevators of $f_{1}$ and $f_{2}$ correspond to $p^{\prime}$, and the node $p$ is at the unique intersection point of the elevator of $C_{i}$ with $L_{0}$. Hence, we find that the total number of tropical morphisms $f$ in $\mathcal{C}_{1}^{k_{1}, k_{2}}$ is

$$
\mathbb{T} N_{n-1}^{\mathrm{red}}\left(l_{0}-1+\sum_{i=1,2} \sum_{j=1}^{k_{i}-1}\left(l_{j}^{i}-1\right),\left\{l_{k_{1}}^{1}-1, l_{k_{1}+1}^{1}, \ldots, l_{\gamma_{1}}^{1}\right\},\left\{l_{k_{2}}^{2}-1, l_{k_{2}+1}^{2}, \ldots, l_{\gamma_{2}}^{2}\right\}\right) .
$$

Given an element $f$ of $\mathcal{C}_{2}^{k_{2}}$, the tropical morphism $\pi \circ f$ induces an element of $\mathbb{T} \mathcal{C}_{\text {red }}\left(\widetilde{L}_{0}^{\prime k_{2}}, \omega_{2}^{1}, \omega_{1}^{2, k_{2}}\right)$. Conversely, any element $f_{1}^{\prime} \cup_{p^{\prime}} f_{2}^{\prime}: C_{1}^{\prime} \cup_{p^{\prime}} C_{2}^{\prime} \rightarrow \mathbb{R}^{n-1}$ of $\mathbb{T} \mathcal{C}_{\text {red }}\left(\widetilde{L}_{0}^{\prime k_{2}}, \omega_{2}^{1}, \omega_{1}^{2, k_{2}}\right)$ has a unique lift $f_{1} \cup_{p} f_{2}: C_{1} \cup_{p} C_{2} \rightarrow \mathbb{R}^{n}$ in $\mathcal{C}_{2}^{k_{2}}$; the elevator of $f_{2}$ corresponds to the node $p^{\prime}$, the elevator of $f_{1}$ corresponds to the unique intersection point of $C_{1}^{\prime}$ and $\widetilde{L}_{k_{0}}^{\prime 1}$, and the node $p$ corresponds to the unique intersection point of the elevator of $f_{2}$ and $L_{0}$. Hence, the total number of tropical morphisms $f$ in $\mathcal{C}_{2}^{k_{2}}$ is

$$
\mathbb{T} N_{n-1}^{\mathrm{red}}\left(l_{0}-1+\sum_{j=1}^{k_{2}-1}\left(l_{j}^{2}-1\right),\left\{\sum_{j=1}^{k_{0}-1}\left(l_{j}^{1}-1\right), l_{k_{0}}^{1}, \ldots, l_{\gamma_{1}}^{1}\right\},\left\{l_{k_{2}}^{2}-1, l_{k_{2}+1}^{2}, \ldots, l_{\gamma_{2}}^{2}\right\}\right) \text {. }
$$


Given an element $f$ of $\mathcal{C}_{3}^{k_{1}, k_{2}}$, the tropical morphism $\pi \circ f$ induces an element of $\mathbb{T} \mathcal{C}_{\text {red }}\left(\mathcal{L}_{0}^{\prime k_{2}}, \omega_{3}^{1, k_{1}}, \omega_{1}^{2, k_{2}}\right)$. Conversely, any element $f_{1}^{\prime} \cup_{p^{\prime}} f_{2}^{\prime}: C_{1}^{\prime} \cup_{p^{\prime}} C_{2}^{\prime} \rightarrow \mathbb{R}^{n-1}$ of $\mathbb{T} \mathcal{C}_{\text {red }}\left(\mathcal{L}_{0}^{\prime k_{2}}, \omega_{3}^{1, k_{1}}, \omega_{1}^{2, k_{2}}\right)$ has a unique lift $f_{1} \cup_{p} f_{2}: C_{1} \cup_{p} C_{2} \rightarrow \mathbb{R}^{n}$ in $\mathcal{C}_{3}^{k_{1}, k_{2}}$. Hence, the total number of tropical morphisms $f$ in $\mathcal{C}_{3}^{k_{1}, k_{2}}$ is

$\mathbb{T} N_{n-1}^{\mathrm{red}}\left(l_{0}+\sum_{j=1}^{k_{2}-1}\left(l_{j}^{2}-1\right),\left\{\sum_{j=1}^{k_{1}-1}\left(l_{j}^{1}-1\right), l_{k_{1}}^{1}-1, l_{k_{1}+1}^{1}, \ldots, l_{\gamma_{1}}^{1}\right\},\left\{l_{k_{2}}^{2}-1, l_{k_{2}+1}^{2}, \ldots, l_{\gamma_{2}}^{2}\right\}\right)$.

Altogether with the induction hypothesis, we conclude that

$$
\mathbb{T} N_{n}^{\mathrm{red}}\left(l_{0},\left\{l_{1}^{1}, \ldots, l_{\gamma_{1}}^{1}\right\},\left\{l_{1}^{2}, \ldots, l_{\gamma_{2}}^{2}\right\}\right)=A B
$$

where

$$
A=\sum_{k_{2}=1}^{\gamma_{2}} N_{1, n-1}\left(2 n-2-\sum_{j=k_{2}}^{\gamma_{2}}\left(l_{j}^{2}-1\right), l_{k_{2}}^{2}-1, l_{k_{2}+1}^{2}, \ldots, l_{\gamma_{2}}^{2}\right)
$$

and

$$
\begin{aligned}
B= & \sum_{k_{1}=k_{0}}^{\gamma_{1}} N_{1, n-1}\left(2 n-2-\sum_{j=k_{1}}^{\gamma_{1}}\left(l_{j}^{1}-1\right), l_{k_{1}}^{1}-1, l_{k_{1}+1}^{1}, \ldots, l_{\gamma_{1}}^{1}\right) \\
& +N_{1, n-1}\left(2 n-2-\sum_{j=1}^{\gamma_{1}}\left(l_{j}^{1}-1\right), \sum_{j=1}^{k_{0}-1}\left(l_{j}^{1}-1\right), l_{k_{0}}^{1}, \ldots, l_{\gamma_{1}}^{1}\right) \\
& +\sum_{k_{1}=1}^{k_{0}-1} N_{1, n-1}\left(2 n-1-\sum_{j=1}^{\gamma_{1}}\left(l_{j}^{1}-1\right), \sum_{j=1}^{k_{1}-1}\left(l_{j}^{1}-1\right), l_{k_{1}}^{1}-1, l_{k_{1}+1}^{1}, \ldots, l_{\gamma_{1}}^{1}\right)
\end{aligned}
$$

Now it follows from Corollary 3.8 that

$$
\begin{aligned}
& A=N_{1, n}\left(2 n-1-\sum_{j=1}^{\gamma_{2}}\left(l_{j}^{2}-1\right), l_{1}^{2}, \ldots, l_{\gamma_{2}}^{2}\right), \\
& B=N_{1, n}\left(2 n-1-\sum_{j=1}^{\gamma_{1}}\left(l_{j}^{1}-1\right), l_{1}^{1}, \ldots, l_{\gamma_{1}}^{1}\right),
\end{aligned}
$$

which completes the proof of the proposition.

Acknowledgments. This work has been supported by the Réseau de Coopération France-Brésil. E.B. is also partially supported by the ANR-09-BLAN-0039-02. 


\section{References}

[BPS08] Berline, N., Plagne, A., Sabbah, C., editors: Géométrie tropicale. Éditions de l'École Polytechnique, Palaiseau (2008); http://www.math.polytechnique.fr/xups/vol08.html.

[Ber08] Bertrand, B.: Real Zeuthen numbers for two lines. Int. Math. Res. Notices 2008, no. 8, art. ID rnn014, 8 pp. Z Zbl 1155.14038 MR 2428148

[BBM] Bertrand, B., Brugallé, E., Mikhalkin, G.: Genus 0 characteristic numbers of the tropical projective plane. arXiv:1105.2004.

[BM] Brugallé, E., Mikhalkin, G.: Floor decompositions of tropical curves in any dimension. In preparation; http://people.math.jussieu.fr/ brugalle/articles/FDn/FDGeneral.pdf.

[BM07] Brugallé, E., Mikhalkin, G.: Enumeration of curves via floor diagrams. C. R. Math. Acad. Sci. Paris 345, 329-334 (2007) Zbl 1124.14047 MR 2359091

[BM08] Brugallé, E., Mikhalkin, G.: Floor decompositions of tropical curves: the planar case. In: Proceedings of 15th Gökova Geometry-Topology Conference, 64-90 (2008) Zbl 1200.14106 MR 2500574

[CH98] Caporaso, L., Harris, J.: Counting plane curves of any genus. Invent. Math. 131, 345392 (1998) Zbl 0934.14040 MR 1608583

[DK00] Degtyarev, A. I., Kharlamov, V. M.: Topological properties of real algebraic varieties: Rokhlin's way. Russian Math. Surveys 55, 735-814 (2000) Zbl 1014.14030 MR 1786731

[EGH00] Eliashberg, Y., Givental, A., Hofer, H.: Introduction to symplectic field theory. Geom. Funct. Anal. 2000, Special Volume, Part II, 560-673 Zbl 0989.81114 MR 1826267

[EG02] Eremenko, A., Gabrielov, A.: Degrees of real Wronski maps. Discrete Comput. Geom. 28, 331-347 (2002) Zbl 1004.14011 MR 1923956

[Ful84] Fulton, W.: Introduction to Intersection Theory in Algebraic Geometry. CBMS Reg. Conf. Ser. Math. 54, Amer. Math. Soc., Providence (1984) Zbl 0913.14001 MR 0735435

[GKM09] Gathmann, A., Kerber, M., Markwig, H.: Tropical fans and the moduli spaces of tropical curves. Compos. Math. 145, 173-195 (2009) Zbl 1169.51021 MR 2480499

[IKS04] Itenberg, I., Kharlamov, V., Shustin, E.: Logarithmic equivalence of Welschinger and Gromov-Witten invariants. Uspekhi Mat. Nauk 59, no. 6, 85-110 (2004) (in Russian); English transl.: Russian Math. Surveys 59, no. 6, 1093-1116 (2004) Zbl 1086.14047 MR 2138469

[IMS07] Itenberg, I., Mikhalkin, G., Shustin, E.: Tropical Algebraic Geometry. Oberwolfach Sem. Ser. 35, Birkhäuser (2007) Zbl 1162.14300 MR 2292729

[KV06] Kock, J., Vainsencher, I.: An Invitation to Quantum Cohomology: Kontsevich's Formula for Rational Plane Curves. Progr. Math. 249, Birkhäuser (2006) Zbl 1114.14001 MR 2262630

[Mik] Mikhalkin, G.: Phase-tropical curves I. Realizability and enumeration. In preparation

[Mik05] Mikhalkin, G.: Enumerative tropical algebraic geometry in $\mathbb{R}^{2}$. J. Amer. Math. Soc. 18, 313-377 (2005) Zbl 1092.14068 MR 2137980

[Mik06] Mikhalkin, G.: Tropical geometry and its applications. In: International Congress of Mathematicians (Madrid, 2006), Vol. II, Eur. Math. Soc., Zürich, 827-852 (2006) Zbl 1103.14034 MR 2275625

[NS06] Nishinou, T., Siebert, B.: Toric degenerations of toric varieties and tropical curves. Duke Math. J. 135, 1-51 (2006) Zbl 1105.14073 MR 2259922

[Rey] Rey, J.-E.: unpublished 
[RGST05] Richter-Gebert, J., Sturmfels, B., Theobald, T.: First steps in tropical geometry. In: Idempotent Mathematics and Mathematical Physics, Contemp. Math. 377, Amer. Math. Soc., Providence, RI, 289-317 (2005) Zbl 1093.14080 MR 2149011

[RTV97] Ronga, F., Tognoli, A., Vust, T.: The number of conics tangent to five given conics: the real case. Rev. Mat. Univ. Complut. Madrid 10,391-421 (1997) Zbl 0921.14036 MR 1605670

[Sot] Sottile, F.: 3264 real conics. http://www.math.tamu.edu/ sottile/research/stories/3264/ index.html

[Sot97] Sottile, F.: Enumerative geometry for the real Grassmannian of lines in projective space. Duke Math. J. 87, 59-85 (1997) Zbl 0986.14033 MR 1440063

[Spe08] Speyer, D.: Tropical linear spaces. SIAM J. Discrete Math. 22, 1527-1558 (2008) Zbl 1191.14076 MR 2448909

[Vak00a] Vakil, R.: Counting curves on rational surfaces. Manuscripta Math. 102, 53-84 (2000) Zbl 0967.14036 MR 1771228

[Vak00b] Vakil, R.: The enumerative geometry of rational and elliptic curves in projective space. J. Reine Angew. Math. 529, 101-153 (2000) Zbl 0970.14029 MR 1799935

[Vak06] Vakil, R.: Schubert induction. Ann. of Math. 164, 489-512 (2006) Zbl 1115.14043 MR 2247966

[We105a] Welschinger, J. Y.: Invariants of real symplectic 4-manifolds and lower bounds in real enumerative geometry. Invent. Math. 162, 195-234 (2005) Zbl 1082.14052 MR 2198329

[Wel05b] Welschinger, J. Y.: Spinor states of real rational curves in real algebraic convex 3-manifolds and enumerative invariants. Duke Math. J. 127, 89-121 (2005) Zbl 1084.14056 MR 2126497 